This item was submitted to Loughborough's Research Repository by the author.

Items in Figshare are protected by copyright, with all rights reserved, unless otherwise indicated.

\title{
Generation of ground vibration boom by high-speed trains
}

PLEASE CITE THE PUBLISHED VERSION

PUBLISHER

(c) V.V. Krylov and Thomas Telford Limited 2001

VERSION

AM (Accepted Manuscript)

LICENCE

CC BY-NC-ND 4.0

REPOSITORY RECORD

Krylov, Victor V.. 2013. "Generation of Ground Vibration Boom by High-speed Trains". figshare. https://hdl.handle.net/2134/12353. 
This item was submitted to Loughborough's Institutional Repository (https://dspace.lboro.ac.uk/) by the author and is made available under the following Creative Commons Licence conditions.

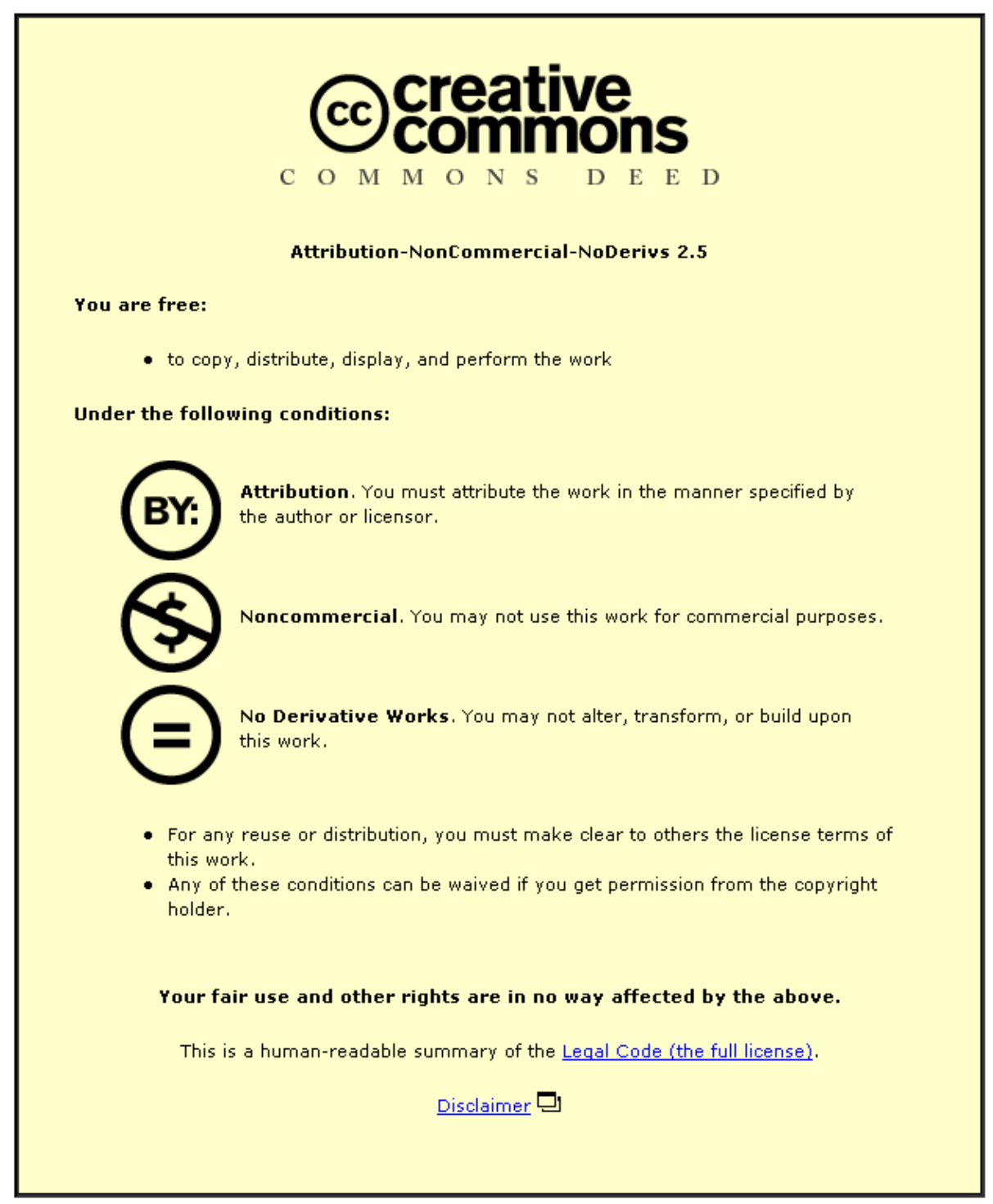

For the full text of this licence, please go to: http://creativecommons.org/licenses/by-nc-nd/2.5/ 
Noise and Vibration from High-Speed Trains (VV Krylov, ed.), Thomas Telford, London, 2001, pp. 251-283

\title{
Chapter 9
}

\section{GENERATION OF GROUND VIBRATION BOOM BY HIGH-SPEED TRAINS}

\author{
V.V. Krylov \\ Department of Civil and Structural Engineering, \\ The Nottingham Trent University, \\ Burton Street, Nottingham NG1 4BU, UK
}

\section{INTRODUCTION}

Railway-generated ground vibrations cause significant disturbance for residents of nearby buildings even when generated by conventional passenger or heavy-freight trains [1,2]. If train speeds increase, the intensity of railway-generated vibrations generally becomes larger. For modern high-speed trains the increase in ground vibration intensity is especially high when train speeds approach certain critical velocities of waves propagating in a track-ground system. The most important are two such critical velocities: the velocity of Rayleigh surface wave in the ground and the minimal phase velocity of bending waves propagating in a track supported by ballast, the latter velocity being referred to as track critical velocity. Both these velocities can be easily exceeded by modern high-speed trains, especially in the case of very soft soil where both critical velocities become very low.

As has been theoretically predicted by the present author [3,4], if a train speed $v$ exceeds the Rayleigh wave velocity $c_{R}$ in supporting soil a ground vibration boom occurs. It is associated with a very large increase in generated ground vibrations, as compared to the case of conventional trains. The phenomenon of ground vibration boom is similar to a sonic boom for aircraft crossing the sound barrier, and its existence has been recently confirmed experimentally [5,6] (see also chapter 11). The measurements have been carried out on behalf of the Swedish Railway Authorities when their West-coast Main Line from Gothenburg to Malmö was opened for the X2000 high-speed train. The speeds achievable by the X2000 train (up to $200 \mathrm{~km} / \mathrm{h}$ ) can be larger than lowest Rayleigh wave velocities in this part of Sweden characterised by very soft ground. In particular, at the location near Ledsgärd the Rayleigh wave velocity in the ground was around $45 \mathrm{~m} / \mathrm{s}$, so the increase in train speed from 140 to $180 \mathrm{~km} / \mathrm{h}$ lead to about 10 times increase in generated ground vibrations [5] (see chapter 11). The above mentioned first observations of ground vibration boom indicate that now one can speak about "supersonic" ("superseismic") or, more precisely, "trans-Rayleigh" trains [7-9]. The increased attention of railway companies and local authorities to ground vibrations associated with high-speed trains stimulated a growing number of theoretical and experimental investigations in this area (see, e.g. [10-13]). 
If train speeds increase further and approach the track critical velocity, then rail deflections due to applied wheel loads may become essentially larger. Possible very large rail deflections around this speed may result even in train derailment, thus representing a serious problem for train and passenger safety [6,14-16]. From the point of view of generating ground vibrations outside the track, these large rail deflections can be responsible for an additional growth of ground vibration amplitudes, as compared to the above mentioned case of ground vibration boom $[7,9,17]$.

In the present paper we review the current status of the theory of ground vibration boom from high-speed trains. Among the problems to be discussed are the quasi-static pressure generation mechanism, effects of Rayleigh wave velocity and track wave resonances on generated ground vibrations, effects of layered geological structure of the ground, and waveguide effects of the embankments. The results of theoretical calculations for TGV and Eurostar high-speed trains travelling along typical tracks are compared with the existing experimental observations.

\section{QUASI-STATIC PRESSURE MECHANISM OF GENERATING GROUND VIBRATIONS}

In what follows, an idealised model of a train comprising $N$ carriages is considered. It is assumed that the train is travelling at speed $v$ along the track with sleeper periodicity $d$ (Fig. 1,a).

Fig. 1 here

One can distinguish several mechanisms of railway-generated ground vibrations which may contribute to the total ground vibration level in different frequency bands. Among these mechanisms one can mention the quasi-static wheel-axle pressure onto the track, the effects of joints in unwelded rails, the unevenness of wheels or rails, and the dynamically induced forces of carriage- and wheel-axle vibrations excited mainly by unevenness of wheels and rails.

Among the above mentioned mechanisms, we consider only the most common one which is present even for ideally flat rails and wheels - a quasi-static pressure of wheel axles onto the track. The quasi-static pressure generation mechanism causes downward deflections of the track beneath each wheel axle (Fig. 1,b). These result in distribution of the axle loads over the sleepers involved in the deflection distance $[3,4,18]$. Thus, each sleeper acts as a dynamic vertical force applied to the ground during the time necessary for a deflection curve to pass over the sleeper. In the framework of the wheel-axle pressure mechanism it is these forces that result in generating ground vibrations by passing trains. As will be demonstrated below, this mechanism is also responsible for railway-generated ground vibration boom. The role of other generation mechanisms is discussed elsewhere [9].

\subsection{Track dynamic properties}

An essential aspect of analysing the above mentioned quasi-static pressure generation mechanism is calculation of the track deflection curve as function of the applied axle load and train speed. One can treat each rail as an Euler - Bernoulli elastic beam of uniform mass $m_{0}$ ( $m_{0}$ includes the contribution of sleeper) lying on a visco-elastic half space $z>0$ and use the following dynamic equation to describe its vertical deflections (see, e.g. [19]): 


$$
E I \partial^{4} w / \partial x^{4}+m_{0} \partial^{2} w / \partial^{2}+2 m_{0} \omega_{b} \partial w / \partial t+\alpha w=T \delta(x-v t)
$$

Here $w$ is the beam deflection magnitude, $E$ and $I$ are Young's modulus and the crosssectional momentum of the beam, $\omega_{b}$ is the circular frequency of damping, $\alpha$ is the proportionality coefficient of the equivalent Winkler elastic foundation modelling the elastic ground, $x$ is the distance along the beam, $T$ is a wheel load applied to each rail and considered as a vertical point force, $v$ is a train speed, and $\delta(x)$ is the Dirac's deltafunction.

It is useful first to discuss free wave propagation in the supported beam without damping, i.e., to analyse equation (1) with $T=0$ and $\omega_{b}=0$. In this case, the substitution of the solution in the form of harmonic bending waves

$$
w=A \exp (i k x-i \omega t)
$$

into (1) gives the following dispersion equation for track waves propagating in the system:

$$
\omega=\left(\alpha+E I k^{4}\right)^{1 / 2} / m_{0}^{1 / 2} .
$$

Here $k$ is the wavenumber of track waves, and $\omega$ is circular frequency. In the quasi-static (long-wave) approximation ( $k=0$ ) the dispersion equation (3) reduces to the well known expression for the so called track on ballast resonance frequency: $\omega_{t b}=\alpha^{1 / 2} / m_{0}^{1 / 2}$. For example, for typical soil and track parameters $\alpha=52.6 \mathrm{MN} / \mathrm{m}^{2}$ [20], and $m_{0}=300 \mathrm{~kg} / \mathrm{m}$ this gives $F_{t b}=\omega_{t b} / 2 \pi=67 \mathrm{~Hz}$. The frequency $F_{t b}$ represents the minimal frequency at which track waves can propagate. It also follows from equation (3) that the frequencydependent velocity of track wave propagation $c=\omega / k$ is determined by the expression

$$
c=\left(\alpha / k^{2}+E I k^{2}\right)^{1 / 2} / m_{0}^{1 / 2},
$$

which shows that at $k=(\alpha / E I)^{1 / 4}$ the velocity $c$ has a minimum

$$
c_{\text {min }}=\left(4 \alpha E I / m_{0}^{2}\right)^{1 / 4} \text {. }
$$

The value $c_{\min }$ is often referred to as track critical velocity. For the above mentioned typical track and ballast parameters and for the value of $E I$ equal to $4.85 \mathrm{MNm}^{2}$ it follows from (5) that $c_{\min }=326 \mathrm{~m} / \mathrm{s}(1174 \mathrm{~km} / \mathrm{h})$, which is much larger than the speeds of the fastest modern trains. However, for very soft soils, e.g. alluvial soils, characterised by very low $\alpha$, the values of $c_{\min }$ can be as low as $60-70 \mathrm{~m} / \mathrm{s}$ and can be easily exceeded by even relatively moderate high-speed trains.

In practice, the value of $c_{\min }$ for a particular location can be estimated using eqn (5) in which the stiffness of equivalent Winkler foundation $\alpha$ is expressed in terms of real elastic moduli of the ground. There are different theoretical models that give such expressions (see, e.g. [16]). Generally, it follows from these models that track critical velocity is normally larger by $10-30 \%$ than Rayleigh wave velocity for the same ground.

The solution of equation (1) with the right-hand side different from zero has different forms for small and large values of time $t$. In the problem under consideration we are 
interested in an "established" solution for large values of $t$ which describes the track deflections being at rest relative to the co-ordinate system moving at train speed $v$ - the so called stationary solution. Obviously, this solution must depend only on the combination $x$ $v t$. Using the notation $p=\beta(x-v t)$, where $\beta=(\alpha / 4 E I)^{1 / 4}$, it is easy to obtain the stationary solution of (1) in the Fourier domain, $W(p)$, (see, e.g. [19]), where $W(p)=\int_{-\infty}^{\infty} w(z) e^{-i p z} d z$.

Taking the inverse Fourier transform of $W(p)$ gives the analytical expressions for $w(x-v t)$ which have different forms depending on whether $v<c_{\min }, v=c_{\min }$ or $v>c_{\min }$. In particular, if train speed $v$ approaches the minimal phase velocity $c_{\min }$ from below, the rail deflection amplitudes $w$ experience a large resonance increase limited by track damping. Assuming for simplicity that there is no damping in the system $\left(\omega_{b}=0\right)$, one can obtain the exact solution for $v<c_{\min }$ in a very simple form [19]:

$$
w(x-v t)=\left(T / 8 E I \beta^{3} \delta\right) \exp (-\beta \delta|x-v t|)[\cos (\beta \eta(x-v t)+(\delta / \eta) \sin (\beta \eta|x-v t|)] \text {, }
$$

where $\delta=\left(1-v^{2} / c^{2}{ }_{\min }\right)^{1 / 2}$ and $\eta=\left(1+v^{2} / c^{2}{ }_{\min }\right)^{1 / 2}$. One can see that, since the factor $\delta=(1$ $\left.-v^{2} / c^{2}{ }_{\text {min }}\right)^{1 / 2}$ is present in the denominator of the expression (6), the track deflection $w$ increases as the train speed approaches the minimal track wave velocity. Note that in the considered example without damping it follows from (6) that $w \rightarrow \infty$ when $v \rightarrow c_{\text {min }}$. The transition of $v$ over $c_{\min }$ can be considered only with taking damping into account. In this case the approximate analytical expressions for $w(x-v t)$ in the three cases, $v<c_{\min }, v=c_{\min }$ or $v>c_{\min }$, can be found [19]. It follows from these expressions that the amplitudes of rail deflections near track wave resonance, $v=c_{\min }$, are determined by the influence of damping. For typical values of damping these amplitudes are by 2 - 3 times larger than the corresponding static values. The corresponding large rail deflections at train speeds approaching track critical velocity may affect the railway line operation result even in train derailment. Different aspects of this problem are now widely investigated (see, e.g. [6,1416]).

\subsection{Forces applied from sleepers to the ground}

To calculate dynamic forces $P(t-x / v)$ applied from sleepers to the ground, e.g. $P(t)$ - for a sleeper located at $x=0$, one should take into account that $P(t)$ is proportional to the track deflection $w(v t)$ and to the sleeper periodicity $d$ :

$$
P(t)=2 \alpha w(v t) d,
$$

where $\alpha$ is a constant of Winkler foundation, and the presence of two rails has been taken into account. It is convenient to exclude $\alpha$ and $d$ from equation (7). To do so one can use integration of the quasi-static equation (1), i.e., with $m_{0} \partial^{2} w / \partial^{2}=0$, over $x$. The integration results in the formula

$$
\alpha w_{\max }^{s t} d N_{\text {eff }}^{s t}=T
$$


which, combined with equation (7), gives the following expression for $P(t)^{1)}$ :

$$
P(t)=2 T\left[w(v t) / w_{\max }^{s t} N_{e f f}^{s t}\right] .
$$

Here index " $s t$ " corresponds to the quasi-static solution of equation (1) (in particular, $w_{\max }{ }^{s t}$ is the maximum value of $w(v t)$ in the quasi-static approximation), and $N_{\text {eff }}^{\text {st }}$ is the effective number of sleepers equalising the applied quasi-static wheel load $T$ :

$$
\sum_{m=-\infty}^{\infty} \frac{T}{N_{\text {eff }}^{s t}} \frac{w(m d)}{w_{\text {max }}^{s t}}=T,
$$

where $m$ denotes a number of a current sleeper. Numerical solution of equation (10) shows that the value of $N_{\text {eff }}^{\text {st }}$ may be approximated with good accuracy by a simple analytical formula $N_{\text {eff }}^{\text {st }}=0.625 \pi / \beta d=0.625 x_{0}{ }^{\text {st }} / d$, where $x_{0}{ }^{s t}=\pi / \beta$ is the effective quasi-static track deflection distance. Using this formula in equation (9) results in the following expression:

$$
P(t)=3.2 T\left[w(v t) / w_{\max }{ }^{s t}\right]\left(d / x_{0}{ }^{s t}\right) \text {. }
$$

As will be shown below, to describe generation of ground vibrations by moving trains one needs to know the frequency spectrum of a force applied from each sleeper to the ground, $P(\omega)$, rather than its time dependence, $P(t)$. Note that, whereas a time-domain solution $P(t)$ has different forms for $v<c_{\min }, v=c_{\min }$ and $v>c_{\min }$ [19], its Fourier representation $P(\omega)$ is described by the same formula for all these cases. Keeping in mind that for $x=0$ the relationship $W(p)=-2 \pi \beta v W(\omega)$ holds, where $\omega=p \beta v$, one can derive the following expression for $P(\omega)$ :

$$
P(\omega)=\frac{-12.8 \frac{T d}{v \pi^{2}}}{\frac{\omega^{4}}{\beta^{4} v^{4}}-4 \frac{\omega^{2}}{c_{\min }{ }^{2} \beta^{2}}-8 i \frac{g \omega}{c_{\min } \beta}+4},
$$

where $g=\left(m_{0} / \alpha\right)^{1 / 2} \omega_{b}$ is a nondimensional damping parameter. Typical forms of the vertical force spectra $P(\omega)$ calculated for a train travelling on very soft soil at speeds $v=20,50$ and $70 \mathrm{~m} / \mathrm{s}$ (corresponding to the cases $v<c_{R}, \quad c_{R}<v<c_{\min }$, and $v>c_{\min }$ respectively) are shown in Fig. 2.

\section{Fig. 2 here}

Calculations were performed for the soft soil using the following parameters of train, track, and soil: $T=50 \mathrm{kN}, d=0.7 \mathrm{~m}, \beta=1.28 \mathrm{~m}^{-1}, c_{R}=45 \mathrm{~m} / \mathrm{s}, c_{\min }=65 \mathrm{~m} / \mathrm{s}$, and $g=0.1$. For relatively low train speeds, i.e., for $v<c_{R}$, the dynamic solution (12) for the force spectrum $P(\omega)$ goes over to the quasi-static one [18]. As train speeds increase and approach or exceed the minimal track wave velocity, the spectra $P(\omega)$ become broader and larger in amplitudes, and a second peak appears at higher frequencies.

1) In the author's earlier papers [7-9], instead of the sleeper periodicity $d$, the sleeper thickness $\Delta d$ has been used erroneously in equations (7) and (8). Fortunately, this did not affect the expression (9) following from (7) and (8) because of the exclusion of $\alpha$ and $d$. The author is grateful to G. Degrande for pointing this out. 
The values of $P(\omega)$ in the model under consideration are limited by track wave damping described by the nondimensional damping parameter $g$. The effect of track damping on the spectra $P(\omega)$ is more pronounced for $v>c_{\text {min }}$. For low train speeds, $v<c_{R}$, the effect of track damping is negligibly small.

\section{GREEN'S FUNCTION OF THE PROBLEM}

As the next step, one has to derive the Green's function for the problem under consideration. It describes ground vibrations generated by individual sleepers which can be regarded as point sources in the low-frequency band.

\subsection{Homogeneous elastic half-space}

We recall that for a homogeneous elastic half space the corresponding Green's function can be derived using the results from the well-known axisymmetric problem for the excitation of an elastic half space by a vertical point force applied to the surface (see, e.g. [21,22]). The solution of this problem, which should satisfy the dynamic equations of elasticity for a homogeneous medium subject to the stress-free boundary conditions on the surface outside the point of force application, gives the corresponding components of the dynamic Green's tensor (or, for simplicity, the Green's function) $G_{z i}$ for an elastic half space. For the problem under consideration, only Rayleigh surface wave contribution (the Rayleigh part of the Green's function) is considered since Rayleigh waves transfer most of the vibration energy to remote locations. For these waves the spectral density of the vertical vibration velocity at the surface of homogeneous half space $(z=0)$ may be written in the form (see also [18])

$$
v_{z}(\rho, \omega)=P(\omega) G_{z z}(\rho, \omega)=P(\omega) D(\omega)(1 / \sqrt{ } \rho) \exp \left(i k_{R} \rho-\gamma k_{R} \rho\right),
$$

where

$$
D(\omega)=(1 / 2 \pi)^{1 / 2}(-i \omega) q k_{R}^{1 / 2} k_{t}^{2} \exp (-i 3 \pi / 4) / \mu F^{\prime}\left(k_{R}\right)
$$

Here $\rho=\left[\left(x-x^{\prime}\right)^{2}+\left(y-y^{\prime}\right)^{2}\right]^{1 / 2}$ is the distance between the source (with current coordinates $x^{\prime}, y^{\prime}$ ) and the point of observation (with the coordinates $x, y$ ), $\omega=2 \pi F$ is a circular frequency, $k_{R}=\omega / c_{R}$ is the wavenumber of a Rayleigh surface wave, $c_{R}$ is the Rayleigh wave velocity, $k_{l}=\omega / c_{l}$ and $k_{t}=\omega / c_{t}$ are the wavenumbers of longitudinal and shear bulk elastic waves, where $c_{l}=\left[(\lambda+2 \mu) / \rho_{0}\right]^{1 / 2}$ and $c_{t}=\left(\mu / \rho_{0}\right)^{1 / 2}$ are longitudinal and shear wave velocities, $\lambda$ and $\mu$ are the elastic Lame' constants, $\rho_{0}$ is mass density of the ground, and $q=\left(k_{R}^{2}-k_{l}^{2}\right)^{1 / 2}$. The factor $F^{\prime}\left(k_{R}\right)$ is a derivative of the Rayleigh determinant

$$
F(k)=\left(2 k^{2}-k_{t}^{2}\right)^{2}-4 k^{2}\left(k^{2}-k_{t}^{2}\right)^{1 / 2}\left(k^{2}-k_{l}^{2}\right)^{1 / 2}
$$

taken at $k=k_{R}$, and $P(\omega)$ is a Fourier transform of $P(t)$ (see equation (12)).

To describe the spectrum for successive passage of two axle loads separated by the distance $a$ (the case of a bogie), $P_{b}(\omega)$, one should use the following relationship between $P_{b}(\omega)$ and $P(\omega)$ [18]: 


$$
P_{b}(\omega)=2 P(\omega) \cos (\omega a / 2 v) .
$$

In writing eqn (13) we have accounted for attenuation in soil by replacing $1 / c_{R}$ in the exponential of the Green's function by the complex value $1 / c_{R}+i \gamma / c_{R}$, where $\gamma=0.001$ 0.1 is a constant describing the "strength" of dissipation of Rayleigh waves in soil [23].

\subsection{Effect of layered ground structure}

To consider the influence of layered geological structure of the ground on generating ground vibrations in a rigorous way, we would have to use the Green's function for a layered elastic half space, instead of that for a homogeneous half space. As a rule, such a function, that contains information about the total complex elastic field generated in a layered half space considered (including different modes of surface waves and modes radiating energy into the bulk (leaky waves)), can not be obtained analytically (see also chapter 10). However, for description of railway-generated ground vibrations, the problem can be simplified by considering an approximate engineering approach which takes into account the effects of layered structure on the amplitudes and phase velocity of only the lowest order surface mode which goes over to a Rayleigh wave in the limit of a homogeneous half space. The propagating modes of higher orders and leaky modes are generated less efficiently by surface forces associated with sleepers.

We recall that in layered media surface waves become dispersive, i.e., their phase velocities $c_{R}$ depend on frequency: $c_{R}=c_{R}(\omega)$. For shear modulus of the ground $\mu$ normally having larger values at larger depths, there may be several surface modes characterised by different phase velocities and cut-off frequencies. As a rule, these velocities increase at lower frequencies associated with deeper penetration of surface wave energy into the ground (see, e.g. [24]). For simplicity, we will assume in further consideration that the Poisson ratio $\sigma$ of the layered ground and the mass density $\rho_{0}$ are constant. Taking the above mentioned into account and starting from the Green's function for a homogeneous half space $G_{z z}(\rho, \omega)$ (see equations (13) and (14)), we will construct its modification $G_{z z}^{L}(\rho, \omega)$ describing approximately the effects of layered medium on generation and propagation of a lowest order surface Rayleigh type mode. It can be shown that such an approximate Green's function can be written in the form [8]:

$$
\begin{aligned}
& v_{z}(\rho, \omega)=P(\omega) G^{L}{ }_{z z}(\rho, \omega)=P(\omega) D^{L}(\omega)(1 / \sqrt{ } \rho) \exp \left(i k_{R}{ }^{L} \rho-\gamma k_{R}{ }^{L} \rho\right), \\
& D^{L}(\omega)=(1 / 2 \pi)^{1 / 2}(-i \omega) q^{L}\left(k_{R}{ }^{L}\right)^{1 / 2}\left(k_{t}{ }^{L}\right)^{2} \exp (-i 3 \pi / 4) / \mu^{L}(\omega) F_{L}{ }^{\prime}\left(k_{R}{ }^{L}\right) .
\end{aligned}
$$

Here $k_{R}{ }^{L}=\omega / c_{R}(\omega)$ is the wavenumber of a lowest order Rayleigh mode propagating with frequency-dependent velocity $c_{R}(\omega)$; terms $k_{l}^{L}=\omega / c_{l}^{L}(\omega)$ and $k_{t}^{L}=\omega / c_{t}^{L}(\omega)$ are "effective" wavenumbers of longitudinal and shear bulk elastic waves at given frequency $\omega$ (these wavenumbers are inversely proportional to the longitudinal $c_{l}{ }^{L}(\omega)$ and shear $c_{t}^{L}(\omega)$ wave velocities averaged over the "effective" depth of Rayleigh wave penetration into the ground which is close to Rayleigh wavelength). In the model under consideration, these velocities 
and the corresponding "effective" shear modulus $\mu^{L}(\omega)$ are expressed in terms of frequencydependent Rayleigh wave velocity $c_{R}(\omega)$ using the well known relations:

$$
\begin{gathered}
c_{R}(\omega) / c_{t}^{L}(\omega)=(0.87+1.12 \sigma) /(1+\sigma), \\
c_{t}^{L}(\omega) / c_{l}^{L}(\omega)=[(1-2 \sigma) / 2(1-\sigma)]^{1 / 2}, \\
\mu^{L}(\omega)=\rho_{0}\left[c_{t}^{L}(\omega)\right]^{2} .
\end{gathered}
$$

The term $q^{L}$ is defined as $q^{L}=\left[\left(k_{R}{ }^{L}\right)^{2}-\left(k_{l}^{L}\right)^{2}\right]^{1 / 2}$, and the factor $F_{L}{ }^{\prime}\left(k_{R}{ }^{L}\right)$ is determined according to the following relationship [24]:

$$
F_{L}^{\prime}\left(k_{R}^{L}\right)=N(\sigma)\left(k_{R}^{L}\right)^{3}
$$

where $N(\sigma)$ is a dimensionless function of the Poisson ratio $\sigma$ (e.g., for $\sigma=0.25$, the function $N(\sigma)$ takes the value -2.3).

The dependence of Rayleigh wave velocity on frequency, $c_{R}(\omega)$, is determined by the particular profile of layered ground, characterised by the dependence of its elastic moduli $\lambda$, $\mu$ and mass density $\rho_{0}$ on vertical coordinate $z$. Figure 3,a shows a widely used simplified model of layered ground consisting of a single top elastic layer with the parameters $\lambda_{1}, \mu_{1}$ and $\rho_{01}$ placed on an elastic half space with the parameters $\lambda_{2}, \mu_{2}$ and $\rho_{02}$. Figure 3,b demonstrates some more complicated layered structures in the assumption that they are characterised mainly by its shear stiffness $\mu$ as a function of $z$.

\section{Fig. 3 here}

For all ground profiles the determination of the velocity $c_{R}(\omega)$ is a complex boundary-value problem which, generally speaking, requires numerical calculation. In the above described engineering approach we consider published values of the wave velocity functions $c_{R}(\omega)$ using where possible their simple analytical approximations. In particular, the frequencydependent Rayleigh wave velocity for layered media characterised by monotonous change of their mechanical parameters with depth (e.g. for simple two-layered systems) can be approximated by the function

$$
c_{R}(\omega)=\left(c_{1}-c_{2}\right) \exp \left(-\alpha^{\prime} \omega / 2 \pi\right)+c_{2},
$$

where $c_{1}$ and $c_{2}$ are values of $c_{R}(\omega)$ for $\omega=0$ and $\omega=\propto$ respectively, the parameter $\alpha^{\text {c }}$ describes "strengths" of dispersion (it depends on the thickness of characteristic layer and on the difference between the elastic moduli in the depth and on the surface of the ground). Figure 4 shows typical functions $c_{R}(\omega)$ calculated according to equation (23) for the value of the parameter $\alpha^{6}=0.1$. Curve Cd1 corresponds to a soft layer $\left(c_{2}=125 \mathrm{~m} / \mathrm{s}\right)$ placed on a stiff ground $\left(c_{1}=200 \mathrm{~m} / \mathrm{s}\right)$. Curve Cd3 describes a stiff layer $\left(c_{2}=200 \mathrm{~m} / \mathrm{s}\right)$ placed on a soft ground $\left(c_{1}=125 \mathrm{~m} / \mathrm{s}\right)$. The value of the parameter $\alpha^{6}=10$ describes the situation when the effect of ground substrate can be neglected and functions $c_{R}(\omega)$ are determined entirely by the upper layers (curves Cd2 and Cd4 - respectively for soft and stiff layers).

\section{Fig. 4 here}


For non-monotonous layered media (see Fig. 3,b), which result in Rayleigh-type mode velocities $c_{R}(\omega)$ being non-monotonous functions of frequency (with maxima or minima at certain frequencies) the simple formula (23) is no longer applicable and other approximate analytical expressions must be sought.

One can expect that the most significant effect of layered structure on generating ground vibrations by high-speed trains is due to the wave phase variations caused by frequencydependent Rayleigh wave velocities, rather than to the changes in wave generation efficiency due to the stratification. In particular, for the considered case of monotonous layered system with soft upper layer, the increase in Rayleigh wave velocities at low frequencies might violate the trans-Rayleigh condition $v>c_{R}$ responsible for generation of very intensive ground vibrations associated with ground vibration boom, thus causing a reduction in the low-frequency components of generated ground vibration spectra.

\section{CALCULATION OF GENERATED GROUND VIBRATIONS}

To calculate ground vibrations generated by a train one needs to take into account the superposition of waves generated by each elementary source (sleeper) activated by wheel axles of all carriages, with the time and space differences between sources (sleepers) being taken into account. Using the Green's function this may be written in the form $[4-9,18]$

$$
v_{z}(x, y, \omega)=\int_{-\infty-\infty}^{\infty} \int_{-\infty}^{\infty} P\left(x^{\prime}, y^{\prime}, \omega\right) G_{z z}^{L}(\rho, \omega) d x^{\prime} d y^{\prime},
$$

where $P\left(x^{\prime}, y^{\prime}, \omega\right)$ describes the space distribution of all load forces acting along the track in the frequency domain. This distribution can be found by taking a Fourier transform of the time and space dependent load forces $P\left(t, x^{\prime}, y^{\prime}=0\right)$ applied from the track to the ground. Note that the function $P\left(t, x^{\prime}, y^{\prime}=0\right)$ does not depend on layered structure of the ground and remains the same for both homogeneous and inhomogeneous half spaces. In the model under consideration, all properties of track and train, which determine generation of ground vibrations, are described by the above mentioned function of load forces $P\left(t, x^{\prime}, y^{\prime}=0\right)$.

\subsection{Vibrations from a single axle load}

It is useful first to consider ground vibrations generated by a single axle load. For a single axle load moving at speed $v$ along the track, the load function has the form $[4,18]$ :

$$
P\left(t, x^{\prime}, y^{\prime}=0\right)=\sum_{m=-\infty}^{\infty} P\left(t-x^{\prime} / v\right) \delta\left(x^{\prime}-m d\right) \delta\left(y^{\prime}\right),
$$

where delta-function $\delta\left(x^{\prime}-m d\right)$ takes the periodic distribution of sleepers into account. Taking the Fourier transform of (25), substituting it into (24) and taking equations (17) and (18) into account results in the following formula for the vertical vibration velocity of Rayleigh waves generated at $z=0, x=0, y=y_{0}$ by a single axle load moving along the track at speed $v$ : 


$$
v_{z}\left(x=0, y=y_{0}, \omega\right)=P(\omega) D^{L}(\omega) \sum_{m=-\infty}^{\infty} \exp \left[i(\omega / v) m d+(i-\gamma)\left(\omega / c_{R}(\omega)\right) \rho_{m}\right] / \sqrt{ } \rho_{m},
$$

where $\rho_{m}=\left[y_{0}^{2}+(m d)^{2}\right]^{1 / 2}$.

\subsection{Vibrations from a complete train}

To take account of all axles and carriages one should use a more complicated load function $[4,18]$ (see also chapter 10 which gives a modified form of this function):

$$
P\left(t, x^{\prime}, y^{\prime}=0\right)=\sum_{m=-\infty}^{\infty} \sum_{n=0}^{N-1} A_{n}\left[P\left(t-\left(x^{\prime}+n L\right) / v\right)+P\left(t-\left(x^{\prime}+M+n L\right) / v\right)\right] \delta\left(x^{\prime}-m d\right) \delta\left(y^{\prime}\right),
$$

where $N$ is the number of carriages, $M$ is the distance between the centres of bogies in each carriage and $L$ is the total carriage length. Dimensionless quantity $A_{n}$ is an amplitude weight-factor to account for different carriage masses. For simplicity we assume all carriage masses to be equal $\left(A_{n}=1\right)$.

Taking the Fourier transform of (27), substituting it into (24) and making simple transformations, we obtain the following expression for the frequency spectra of vertical vibrations at $z=0, x=0$ and $y=y_{0}$ generated by a moving train [4,7-9].

$$
\begin{aligned}
v_{Z}\left(x=0, y=y_{0}, \omega\right)= & P(\omega) D^{L}(\omega) \sum_{m=-\infty}^{\infty} \sum_{n=0}^{N-1}\left[\exp \left(-\gamma \omega \rho_{m} / c_{R}(\omega)\right) / \sqrt{ } \rho_{m}\right][1+\exp (i M \omega / v)] . \\
& \exp \left(i(\omega / v)(m d+n L)+i\left(\omega / c_{R}(\omega)\right) \rho_{m}\right) .
\end{aligned}
$$

Note that the expressions (26) and (28) are applicable to trains moving at arbitrary speeds. In particular, for trains travelling at conventional speeds $\left(v<c_{R}\right)$ they describe peaks in ground vibration spectra corresponding to the well known sleeper passage frequencies and other train-speed dependent combination frequencies [18]. Indeed, the peaks correspond to the frequencies determined by the condition $(\omega / v)(s d+q L)=2 \pi l$, where $s, q, l=1,2,3, \ldots$ Obviously, $q=0$ corresponds to the passage frequencies $f_{p} s=(v / d) l$. Other more frequent maxima are determined either by the carriage length $L \quad(s=0)$ or by a combination of both parameters (for $q \neq 0, s \neq 0$ ) (for experimental validation of these predictions for high-speed trains travelling at speeds below the Rayleigh wave velocity see chapter 10).

\section{TRANS-RAYLEIGH TRAINS}

\subsection{General discussion}

For "trans-Rayleigh trains", i.e., trains travelling at speeds higher than Rayleigh wave velocity in the ground, a separate analytical treatment can be done to elucidate the special features of the problem $[4,9]$. It is easy to show that maximum radiation of ground vibrations takes place if the train speed $v$ and the frequency-dependent Rayleigh wave velocity $c_{R}(\omega)$ satisfy the following condition: 


$$
\cos \Theta=1 / K=c_{R}(\omega) / v
$$

where $\Theta$ is the observation angle. This relationship implies that elastic surface waves radiated by all sleepers activated by moving loads are combined in phase at the point of observation and, as a result, a ground vibration boom takes place. Since the observation angle $\Theta$ must be real ( $\cos \Theta \leq 1)$, the value of $K=v / c_{R}(\omega)$ should be larger than 1, i.e., the train speed $v$ should be larger than Rayleigh wave velocity $c_{R}(\omega)$. In this case ground vibrations are generated as quasi-plane Rayleigh surface waves symmetrically propagating at angles $\Theta$ with respect to the track, and with amplitudes much larger than in the case of "sub-Rayleigh trains". The formation of a ground vibration boom is illustrated in Fig. 5 showing the spatial distributions of the ground surface vertical displacements generated at a chosen frequency component by a single axle load moving at different speeds over a small part of a track consisting of 10 sleepers.

\section{Fig. 5 here}

The results are shown for sub-Rayleigh speed - (a) and trans-Rayleigh speed - (b). It is seen that in the first case the generated ground vibrations propagate almost in all directions, whereas in the second case they are concentrated around the angles $\Theta$ determined by equation (29).

The amplitudes of railway-generated ground vibrations for $v>c_{R}$ are determined by two features. The first one is that under this condition the surface waves radiated by different sleepers are combined in phase. Therefore, an increase by the number of effectively radiating sleepers of the track, i.e., about 200 times for typical values of $\gamma=0.05$, can be expected compared to the average vibration level for conventional trains. The second feature is the dependence of the function $P(\omega)$, determined by equation (12), on train speed $v$. The analysis shows that function $P(v, \omega)$ provides an average increase of about 10 times for the increase of train speed $v$ from $13.88 \mathrm{~m} / \mathrm{s}(50 \mathrm{~km} / \mathrm{h})$ to $138.8 \mathrm{~m} / \mathrm{s}(500 \mathrm{~km} / \mathrm{h})$. Thus, a total increase of ground vibration amplitudes by $1000-2000$ times (60-66 dB) can be expected in the case of trans-Rayleigh trains for such increase in train speeds.

It also follows from equations (26) and (28) that the amplitudes of generated ground vibration boom do not depend on the sleeper periodicity $d$. They are determined only by the track distance considered [4,9]. Note that this conclusion remains valid also in the limiting case $d \rightarrow 0$. This means that radiation of ground vibration boom by trans-Rayleigh trains may take place also on tracks without sleepers, i.e. on slab tracks. However, for conventional low-speed trains $\left(v<<c_{R}\right)$, the exponential functions inside the sums in (26) and (28) oscillate quickly as $d \rightarrow 0$, and the sums themselves become close to zero, indicating that ground vibrations in the form of waves are not generated. This agrees with the well known result of the elasticity theory [25] indicating that, for loads moving along a free surface of an elastic half space at speed $v<c_{R}$, radiated waves do not appear (only localised quasi-static fields can accompany the moving load). Thus, the presence of sleepers is essential for generating ground vibrations by conventional trains due to the mechanism of quasi-static pressure considered here.

If train speed increases even further, then the second critical velocity, $\mathrm{c}_{\min }$, makes its contribution to the amplitudes of generated ground vibrations - through the function $P(\omega)$. However, since this function can increase by only 2-3 times for realistic values of track wave dissipation (see section 2.2), its effect on generated vibrations may result only in the increase 
of amplitude by the same amount. It is therefore less important for generating ground vibrations outside the track than the first critical velocity, $c_{R}$.

\subsection{Ground vibrations from TGV or Eurostar trains}

Numerical calculations of ground vibrations generated by high-speed trains can be carried out according to equations (26) or (28) for different values of train speed, different parameters characterising Rayleigh wave dispersion in layered ground, and for different geometrical and physical parameters of both track and train. For relatively short 5-carriage trains it is normally sufficient to carry out the summation over $m$ in equations (26) and (28) from $m=-$ 150 to $m=150$, the corresponding length of track being greater than the total train length $N L$ and the attenuation distance of Rayleigh waves at the frequency band considered. In many practical situations, the frequency-dependent Rayleigh wave velocity for layered media can be approximated by equation (23).

Figure 6 shows surface graphs of the ground vibration spectra (in linear units, relative to the reference level of $10^{-9} \mathrm{~m} / \mathrm{s}$ ) generated by a single axle load $2 T=200 \mathrm{kN}$ moving at speeds ranging from $10 \mathrm{~m} / \mathrm{s}$ to $320 \mathrm{~m} / \mathrm{s}$ for homogeneous (a) and layered (b) ground.

\section{Fig. 6 here}

The results are given for the frequency band $0-50 \mathrm{~Hz}$ and for the value of ground attenuation $\gamma=0.05$. The Poisson's ratio of soil was set at 0.25 , and the mass density $\rho_{0}$ was $2000 \mathrm{~kg} / \mathrm{m}^{3}$. Units of calculation were $\Delta v=10 \mathrm{~m} / \mathrm{s}$ and $\Delta F=1 \mathrm{~Hz}$. Values of the parameter $\alpha^{6}$ in equation (23) corresponding to homogeneous and layered grounds were 10 and 0.1 respectively. Other parameters were: $c_{\min }=326 \mathrm{~m} / \mathrm{s}, g=0.1, \beta=1.28 \mathrm{~m}^{-1}, y_{0}=30$ $m$, where $y_{0}$ is the distance from the track to the observation point.

One can see that with the increase of train speed the ground vibration level generally grows. For relatively low train speeds, the peaks corresponding to the train passage frequencies are almost invisible because of the huge increase of ground vibration level in the trans-Rayleigh range $\left(v \geq c_{R}(\omega)\right)$. This increase goes even further for train speeds approaching the minimal track wave velocity $\left(c_{\min }=326 \mathrm{~m} / \mathrm{s}\right.$ in this example). Comparison of Figures 6,a and 6,b shows that the effect of a two-layered structure results in decrease of generated vibrations at low frequencies.

Figure 7 illustrates the ground vibration spectra (in $\mathrm{dB}$, relative to the reference level of $10^{-9} \mathrm{~m} / \mathrm{s}$ ) generated by complete TGV or Eurostar trains travelling on homogeneous ground for both sub-Rayleigh and trans-Rayleigh train speeds (respectively $v=50 \mathrm{~km} / \mathrm{h}$ - curve $\mathrm{Vz1}$ and $v=500 \mathrm{~km} / \mathrm{h}$-curve Vz2), and for layered ground the same train speeds (curves $\mathrm{Vz3}$ and $\mathrm{Vz} 4$ respectively).

\section{Fig. 7 here}

The train consists of $N=5$ equal carriages with the parameters $L=18.9 \mathrm{~m}$ and $M=15.9 \mathrm{~m}$. Since the bogies of TGV and Eurostar trains have a wheel spacing of $3 \mathrm{~m}$ and are placed between carriage ends, i.e., they are shared between two neighbouring carriages, to use equation (28) one should consider each carriage as having one-axle bogies ( $a=0$ ) separated by the distance $M=15.9 \mathrm{~m}$. Other parameters of track and ground used in calculations are the same as in Figure 6. One can see that for homogeneous ground (curves Vz1 and Vz2) the averaged ground vibration level from a train moving at trans-Rayleigh speed $500 \mathrm{~km} / \mathrm{h}$ 
$(138.8 \mathrm{~m} / \mathrm{s})$ is by approximately $70 \mathrm{~dB}$ higher than from a train travelling at speed $50 \mathrm{~km} / \mathrm{h}$ $(13.8 \mathrm{~m} / \mathrm{s})$. Including the effect of layered ground, however, results in decrease of ground vibration level from a trans-Rayleigh train at low frequencies (curve Vz4). Note, that for trains travelling at low speeds the effect of layered ground structure is small (curves Vz1 and Vz3 are almost indistinguishable).

Calculated ground vibration frequency spectra $v_{z}(f)$ (in $\mathrm{dB}$, versus the reference level of $10^{9} \mathrm{~m} / \mathrm{s}$ ) generated by complete TGV or Eurostar trains travelling on a very soft homogeneous ground at different speeds are shown in Fig. 8 for three values of train speed: $v=20,50$ and $70 \mathrm{~m} / \mathrm{s}$ (curves V1, V2 and V3 respectively).

\section{Fig. 8 here}

The parameters used in calculations were: $T=100 \mathrm{kN}, \gamma=0.05, \beta=1.28 \mathrm{~m}^{-1}, d=0.7 \mathrm{~m}, c_{R}$ $=45 \mathrm{~m} / \mathrm{s}, c_{\min }=65 \mathrm{~m} / \mathrm{s}, g=0.1$ and $y_{0}=30 \mathrm{~m}$. One can see that for the trans-Rayleigh train speed of $50 \mathrm{~m} / \mathrm{s}$ corresponding to the case $c_{R}<v<c_{\min }$ the overall level of generated ground vibrations is much higher than for sub-Rayleigh train speed of $20 \mathrm{~m} / \mathrm{s}$. For train speed of $70 \mathrm{~m} / \mathrm{s}$ exceeding the value of track critical velocity, $c_{\min }$, a significant increase takes place at higher frequencies of generated ground vibration spectra. However, since the amplitudes of high-frequency components are generally low, the overall increase due to the track wave resonance is not very large in comparison with the one associated with ground vibration boom.

It is interesting to compare the above described theory with the recent observations made on the high-speed railway line from Gothenburg to Malmö, near Ledsgärd (see the Introduction). For a very rough estimate, the layered structure of the ground can be ignored, and the same parameters of TGV trains can be used (instead of X2000 train parameters). Calculations of the vertical ground vibration velocity averaged over the frequency range 0-50 $\mathrm{Hz}$ have been carried out (this roughly corresponds to a peak level of vibration velocity used in the observations). We also used the reported low value of Rayleigh wave velocity in the ground $\left(c_{R}=45 \mathrm{~m} / \mathrm{s}\right)$, assuming that the Poisson ratio of the ground $\sigma$ was 0.25 . To facilitate the comparison of the predicted increase in ground vibration level with the observed one the amplitudes of generated ground vibrations were calculated in linear units $(\mathrm{m} / \mathrm{s})$.

The resulting amplitudes as functions of train speed are shown in Fig. 9 for two values of track critical velocity $-c_{\min }=65 \mathrm{~m} / \mathrm{s}$ (curve V1) and $c_{\min }=10000 \mathrm{~m} / \mathrm{s}$ (curve V2) (the latter very large value of $c_{\min }$ describes the hypothetical case when track dynamics effects can be completely ignored).

\section{Fig. 9 here}

One can see that in both cases the predicted amplitudes of the peak vertical velocity of generated ground vibrations change from $2 \cdot 10^{-5} \mathrm{~m} / \mathrm{s}$ at $v=140 \mathrm{~km} / \mathrm{h}(38.8 \mathrm{~m} / \mathrm{s})$ to $16 \cdot 10^{-5}$ $\mathrm{m} / \mathrm{s}$ at $v=180 \mathrm{~km} / \mathrm{h}(50 \mathrm{~m} / \mathrm{s})$. Thus, the estimated 8 times increase in ground vibration level following from the above theory for the given train speeds and Rayleigh wave velocity is in reasonable agreement with the 10 times increase recently observed experimentally for the Swedish high-speed railway line built on the soft ground [5].

According to the conclusions of section 3.2, if one had taken into account the actual ground stratification at Ledsgärd [6] (which can be approximated by a non-monotonous fourlayered system, with the "slowest" layer being positioned beneath the top layer), the predicted values of peak vibration velocity would be roughly the same. The only difference could be expected in the form of generated ground vibration spectrum which would have 
lower values at low and high frequencies, in agreement with the Rayleigh wave velocity on the site having a minimum at certain frequency around $4 \mathrm{~Hz}$.

If we assume that a train speed increases further and approaches or exceeds the track critical velocity $\left(c_{\min }=65 \mathrm{~m} / \mathrm{s}\right)$, then the comparison of curves V1 and V2 shows that the level of generated ground vibrations also becomes larger (by approximately 1.5-2 times, as compared to the case of absence of track dynamics effects). This increase is not as large as in the case of ground vibration boom. However, since it occurs in combination with the latter, this gives a noticeable amplification of the resulting ground vibration impact.

\subsection{High-speed trains travelling underground}

For high-speed underground trains the contribution of bulk shear and longitudinal elastic waves (S- and P-waves respectively) is often more essential than that of Rayleigh waves considered in the previous sections [26]. Obviously, radiated S- and P-waves can also be significantly amplified if train speeds are high enough and the conditions $v>c_{t}$ or even $v$ $>c_{l}$ hold, in addition to the trans-Rayleigh condition $v>c_{R}$ considered so far (we recall that $c_{R}<c_{t}<c_{l}$.). In such cases one can expect that these waves will be radiated into the ground as conical Mach waves propagating at the angles $\Theta_{t}=\arccos \left(c_{t} / v\right)$ and $\Theta_{l}=$ $\arccos \left(c_{l} / v\right)$ relative to the track, in addition to the relatively low-amplitude Rayleigh waves radiated as quasi-plane waves along the surface at the angles $\Theta=\arccos \left(c_{R} / v\right)$. The most likely contribution to the ground vibration boom from underground trains might be that of radiated S-waves since their velocity $c_{t}$, being only about $10 \%$ higher than the velocity of Rayleigh waves, can be more easily exceeded by moving trains than the velocity of longitudinal (compressive) waves $c_{l}$. In the presence of layered structure in the ground around the tunnel, the S-waves initially radiated at the angles $\Theta_{t}=\arccos \left(c_{t} / v\right)$ relative to the track, may experience total internal reflection from the lower layers characterised by higher shear wave velocities and return to the surface. Repeated reflections from the ground surface and from lower layers may cause a waveguide propagation of S- and P-waves which will affect the total vibration field in relatively remote locations.

In comparison with surface trains, the case of underground trains is more difficult for theoretical description, partly because of the influence of the tunnel geometry making the problem of constructing the corresponding Green's function extremely complex. We recall that physical meaning of the Green's function for the problem under consideration is that it describes ground vibrations generated by an individual sleeper which may be regarded as a point source in the low-frequency band. In the case of underground trains, i.e., for sleepers placed on the bottom of a tunnel, bulk acoustic waves usually make a major contribution to the ground vibration field near the surface (especially if the tunnel is deep enough), in contrast to the case of above-ground trains where Rayleigh surface acoustic waves prevail.

The approximate analytical approach described here considers the problem in the lowfrequency approximation, i.e., the characteristic wave-lengths of generated bulk acoustic waves in the ground are assumed to be essentially larger than the diameter of the tunnel (Fig. 10). For simplicity, we only consider the case of homogeneous ground.

\section{Fig.10 here}

In the above mentioned low-frequency approximation the formal expression for the vertical component of the particle velocity of ground vibrations generated on the ground surface by trains travelling underground may be written as follows [26]: 


$$
v_{z}(x, y, \omega)=\int_{-\infty-\infty}^{\infty} \int_{-\infty}^{\infty} P\left(x^{\prime}, y^{\prime}, z^{\prime}, \omega\right) G_{z z}(r, \omega) d x^{\prime} d y^{\prime} d z^{\prime}
$$

Here $G_{z z}(r, \omega)$ is the correspondent component of the elastic Green's tensor (Green's function) satisfying the boundary conditions on the ground surface and describing the vertical component of the particle vibration velocity due to a vertical point force located on the bottom of the tunnel, $r=\left[\left(x-x^{\prime}\right)^{2}+\left(y-y^{\prime}\right)^{2}+\left(z-z^{\prime}\right)^{2}\right]^{1 / 2}$ is the distance from the current elementary source to the observation point, and $P\left(x^{\prime}, y,{ }^{\prime}, z^{\prime}, \omega\right)$ describes the Fourier transform of the total distribution of vertical load forces along the underground track.

For low frequencies of radiated waves (around 10-15 Hz) the approximate expression for the far-field Green's function $G_{z z}(r, \omega)$ at the observation point taken on the surface $(z=0)$ may be written in the zero approximation versus the tunnel diameter $a$ (i.e. independent of a), with only bulk elastic waves being taken into account [26]:

$$
\left.\left.G_{z z}(r, \omega)\right|_{z=0}=\left(i \omega / 4 \pi \rho_{0} r\right)\left[e^{i(\omega / c}\right) r\left(1+R_{l}(\omega, \varphi)\right) \cos ^{2}(\varphi)-e_{t}^{i(\omega / c) r}\left(1+R_{t}(\omega, \varphi)\right) \sin ^{2}(\varphi)\right] .
$$

Here terms with the exponents $e^{i(\omega / c) r}$ and $e_{t}^{i(\omega / c) r}$ describe contributions of radiated longitudinal and shear bulk waves, $\left.R_{l}(\omega, \varphi)\right)$ and $R_{t}(\omega, \varphi)$ are the corresponding reflection coefficients from the surface for the incident longitudinal and shear waves respectively (note that each of these coefficients takes account of both waves reflected from the surface longitudinal and shear), and $\varphi$ is the observation angle relative to the vertical direction $\left(\cos \varphi=\left(z-z^{\prime}\right) / r\right)$. The dependence on frequency in $\left.R_{l}(\omega, \varphi)\right)$ and $R_{t}(\omega, \varphi)$ takes account of impedance load resulting from the influence of buildings or other engineering structures on the surface. In what follows we assume, without loss of generality, that $\left.R_{l}(\omega, \varphi)\right)=0$ and $R_{t}(\omega, \varphi)=0$, i.e. consider that all the energy of radiated waves is absorbed by the structure.

The load-force distribution along underground tracks may be written in the form similar to that for the surface tracks (see equation (27)). For a complete train moving at speed $v$ along the track lying underground at the depth $H$ this distribution has the form [26]

$$
P\left(t, x^{\prime}, y^{\prime}, z^{\prime}\right)=\sum_{m=-\infty}^{\infty} \sum_{n=0}^{N-1} A_{n}\left[P\left(t-\left(x^{\prime}+n L\right) / v\right)+P\left(t-\left(x^{\prime}+M+n L\right) / v\right)\right] \delta\left(x^{\prime}-m d\right) \delta\left(y^{\prime}\right) \delta\left(z^{\prime}-H\right) \text {. }
$$

All other notations in equation (32) are the same as in the previous sections.

Taking the Fourier transform of (32) and substituting it into equation (30) with account of (31), one can obtain the following expression for the frequency spectra of vertical vibrations at $z=0, x=0$ and $y=y_{0}$ generated by an underground train [26]:

$$
\begin{gathered}
v_{Z}\left(0, y_{0}, \omega\right)=\left[i \omega P(\omega) / 4 \pi \rho_{0}\right] \sum_{m=-\infty}^{\infty} \sum_{n=0}^{N-1}[1+\exp (i M \omega / v)] \exp [i(\omega / v)(m d+n L)] x \\
\left(1 / r_{m}\right)\left\{\exp \left[\left(-\gamma_{l} \omega r_{m} / c_{l}\right)+i\left(\omega / c_{l}\right) r_{m}\right] \cos ^{2}\left(\varphi_{m}\right)-\exp \left[\left(-\gamma_{t} \omega r_{m} / c_{t}\right)+i\left(\omega / c_{t}\right) r_{m}\right] \sin ^{2}\left(\varphi_{m}\right)\right\} .
\end{gathered}
$$


Here $r_{m}=\left[y_{0}{ }^{2}+(m d)^{2}+H^{2}\right]^{1 / 2}, \cos \left(\varphi_{m}\right)=H / r_{m}$, and $P(\omega)$ is a Fourier transform of $P(t)$ described by equation (12). In writing (33) we have taken into account attenuation in soil by replacing $1 / c_{l}$ and $1 / c_{t}$ in the exponentials by the complex values $1 / c_{l}+i \gamma_{l} / c_{l}$ and $1 / c_{t}+$ $i \gamma_{t} / c_{t}$, where $\gamma_{l, t}<<1$ are the constants describing "strength" of dissipation of longitudinal and shear waves in the soil. To generalise equation (33) to describe the action of two axle loads separated by the distance $a$ (the case of a bogie) one should replace $P(\omega)$ by $P_{b}(\omega)$, according to (16).

It follows from equation (33) that, similarly to the case of surface trains (see section 4.2), the spectrum of ground vibrations from underground trains is quasi-discrete, with the maxima at frequencies determined by the condition $(\omega / v)(s d+q L)=2 \pi$, where $s, q, l$ $=1,2,3, \ldots$

Calculations according to equation (33) show that for underground trains travelling at conventional speeds the main contribution to the vertical component $v_{z}$ of the total ground vibration field at the ground surface is usually due to the radiated shear bulk waves rather than to the longitudinal bulk waves.

In the case of high-speed underground trains, the analysis of equation (33) shows that it has two maxima for the values of observation angles satisfying the conditions: $\cos \Theta_{t}=c_{t} / v$ - for radiated shear waves, and $\cos \Theta_{l}=c_{l} / v$ - for radiated longitudinal waves. Since $\cos \Theta_{t}$ and $\cos \Theta_{l}$ must be less than 1 it follows from these conditions that the maxima can be achieved if the train speeds are high enough and the conditions $v>c_{t}$ or even $v>c_{l}$ hold. In such cases the corresponding waves waves are radiated into the ground as conical waves propagating at the angles $\Theta_{t}=\arccos \left(c_{t} / v\right)$ and $\Theta_{l}=\arccos \left(c_{l} / v\right)$ relative to the track.

The results of calculations of the amplitudes of generated ground vibrations at frequency

$15 \mathrm{~Hz}$ (in $\mathrm{dB}$ re $10^{-9} \mathrm{~m} / \mathrm{s}$ ) as functions of the tunnel depth $H$ for $Y_{0}=30 \mathrm{~m}$ are shown in Fig. 11 for two values of train speed: $v=13.8 \mathrm{~m} / \mathrm{s}$ (conventional speed) and $\mathrm{v}=80 \mathrm{~m} / \mathrm{s}$ (transshear speed for the ground considered). The vertical components of ground vibration velocity on the ground surface are indicated as Vz1 and Vz2 respectively for $\mathrm{v}=13.8 \mathrm{~m} / \mathrm{s}$ and $\mathrm{v}=80 \mathrm{~m} / \mathrm{s}$. The corresponding separate contributions of longitudinal and shear elastic waves are shown as VzL1, VzS1 and VzL2, VzS2.

\section{Fig. 11 here}

The parameters of a TGV high-speed train with $N=5$ carriages are the same as in the previous examples. Other parameters are the following: the elastic parameters of the ground are $c_{t}=76 \mathrm{~m} / \mathrm{s}, \quad c_{1}=129 \mathrm{~m} / \mathrm{s}$, and $\mathrm{C}_{\mathrm{R}}=70 \mathrm{~m} / \mathrm{s}$ (corresponding to the Poisson's ratio $\sigma$ $=0.25$ ), the mass density of soil $\rho_{0}$ is $2000 \mathrm{~kg} / \mathrm{m}^{3}$, and the wheel load $T$ is $100 \mathrm{kN}$. The soil attenuation parameters $\gamma_{1, t}$ were chosen as 0.05 , and the effect of track wave velocity was neglected.

It is seen from Fig. 11 that ground vibrations generated by an underground train travelling at speed $v$ larger than shear wave velocity in the ground $c_{t}$ are essentially larger than those generated by the same train moving at conventional speed (the speed-related amplification of the total ground vibration field varies from about $50 \mathrm{~dB}$ to $20 \mathrm{~dB}$ for the tunnel depth $H$ changing from $2 \mathrm{~m}$ to $100 \mathrm{~m}$ ). For practical values of $H$ (less than $60-70 \mathrm{~m}$ ) the contributions of shear waves for the given Poisson's ratio is essentially higher than the contribution of longitudinal waves. For larger depths, the contributions of shear and longitudinal waves first become comparable with each other, causing an oscillatory behaviour of the resulting field versus $H$, and then longitudinal waves prevail. Note in this connection that the difference between the ground vibration levels corresponding to the 
contributions of longitudinal waves at $v=13.8 \mathrm{~m} / \mathrm{s}$ and $v=80 \mathrm{~m} / \mathrm{s}$ (about $20 \mathrm{~dB}$, or 10 times) is attributed entirely to the effect of the function $P(v, \omega)$ determined by equation (12) (see also section 5.1) since no ground vibration boom is experienced with regard to longitudinal waves in the case considered. Therefore, the observed additional increase of up to $30 \mathrm{~dB}$ in the resulting ground vibration field is associated with the ground vibration boom for radiated shear waves.

Generated ground vibrations (in $\mathrm{dB}$ re $10^{-9} \mathrm{~m} / \mathrm{s}$ ) as functions of the observation distance from the track $Y_{0}$ for $H=30 \mathrm{~m}$ at frequency $15 \mathrm{~Hz}$ are shown in Fig. 12 for the same values of train speed ( $\mathrm{v}=13,8 \mathrm{~m} / \mathrm{s}$ and $80 \mathrm{~m} / \mathrm{s})$ and the same train, track and ground parameters curves Vz01 and Vz02 respectively. One can see that in both cases the fields decrease with the distance $\mathrm{Y}_{0}$, especially in the case of ground vibration boom in respect to shear waves.

\section{Fig. 12 here}

The results of calculations of total ground vibration spectra generated by the same TGV train comprising five carriages $(N=5)$ and travelling underground at speeds $\mathrm{v}=13,8 \mathrm{~m} / \mathrm{s}$ and $80 \mathrm{~m} / \mathrm{s}$ are shown on Fig. 13 (curves $V z 1$ and $V z 2$ respectively). The train, track and ground parameters are the same as in Figs.11 and 12, $H=30 \mathrm{~m}$ and $Y_{0}=30 \mathrm{~m}$. For comparison, on the same figure the spectra are shown generated by the same train travelling above the ground for $Y_{0}=30 \mathrm{~m}$ (curves $V z 1 R$ and $V z 2 R$ ). In the latter case, which was widely discussed in the previous sections, the Rayleigh wave contribution prevails.

\section{Fig. 13 here}

One can see that in the case of ground vibration boom which is present at $v=80 \mathrm{~m} / \mathrm{s}$ for both underground and surface trains, a very large increase in generated ground vibrations is observed, albeit for an underground train this increase is less pronounced, especially at higher frequencies. One can see that the shapes of ground vibration spectra for underground and above ground trains travelling at the same speeds are very similar. This implies that the shapes of ground vibration spectra are determined mainly by track and train geometrical parameters rather than by the tunnel depth and consequently by types of predominantly generated elastic waves.

We remind the reader that the low-frequency approximation used in this section to describe ground vibrations from underground trains is inaccurate for frequencies higher than 10-15 Hz. To improve the situation, one can take into account the next term in the series expansion of the Green's function for the problem under consideration [27]. This term is proportional to the product of tunnel diameter and characteristic wavenumber of radiated ground vibrations.

\subsection{Waveguide effects of the embankments on generated ground vibration fields}

Lots of interesting phenomena can be expected when high-speed railway lines are built on the embankments. In particular, one can mention possible waveguide effects of the embankments on generated ground vibration boom which have been first discussed in Ref. [4]. The possibility of the embankments acting as waveguides for generated ground vibrations is 
closely related to the fact that Rayleigh surface waves associated with ground vibration boom are usually radiated at small angles relative to the track. This is why a dominant part of the radiated energy can be expected to be trapped and dissipated within the embankment itself, without significant leakage to the area outside. In what follows we briefly discuss the process of generating ground vibration boom by high-speed trains travelling along the tops of the embankments.

The effect of railway embankments on generated ground vibrations can be considered by means of construction of the specific Green's function for an elastic half space with an embankment. Such a Green's function must take into account the internal reflections of generated surface Rayleigh waves from the geometric boundaries between the embankment's top flat and side slop surfaces, and between side slop surfaces and the low ground. For simplicity, we assume that elastic properties of the embankment are the same as those of the supporting ground. Also, we consider only reflections from the boundaries between the embankment's top flat surface having the width $2 W$ and side slop surfaces, assuming that side slop surfaces transfer smoothly to the ground, so that no additional reflections take place.

The theory of Rayleigh wave reflection from the boundary between two surfaces intersecting at obtuse angle $\Theta$ has been earlier developed by the present author (see, e.g. [24], p. 277). The corresponding expression for the Rayleigh wave reflection coefficient has the form

$$
R(\alpha)=\frac{i k_{t}{ }^{4}\left(k_{R}{ }^{2}-k_{t}{ }^{2}\right)^{1 / 2}}{2 k_{R}{ }^{2} F^{\prime}\left(k_{R}\right)} \beta \frac{1}{\cos \alpha}\left(1-4 \frac{k_{R}{ }^{2}}{k_{t}{ }^{2}} \sin ^{2} \alpha\right),
$$

where $\beta=\pi-\Theta$, and $\alpha$ is the incidence angle of Rayleigh wave versus the normal to the boundary between the two surfaces; other notations are the same as in the previous sections. It follows from (34) that $R$ is independent of frequency and is defined only by the angle $\beta$ (measured in radians), Poisson's ratio of the ground $\sigma$ and the angle of incidence $\alpha$. Note that the expression (34) has been derived for relatively small $\beta$ and $\alpha$. Therefore, it becomes invalid for $\alpha$ approaching $\pi / 2$, where $|R(\alpha)|$ becomes larger than 1 . To extrapolate $|R(\alpha)|$ for arbitrary $\alpha$, we introduce a truncated reflection coefficient $R_{t}(\alpha)$ in the following way:

$$
R_{t}(\alpha)=R(\alpha) \Phi(1-|R(\alpha)|)+i \Phi(|R(\alpha)|-1)
$$

where $\Phi(\mathrm{z})$ is a Heaviside step function. The behaviour of the amplitude and phase of $R_{t}(\alpha)$ is shown in Fig. 14 for $\beta=30^{\circ}$ and Poisson's ratio $\sigma=0.25$.

\section{Fig. 14 here}

For the problem under consideration, one can use the above mentioned reflection coefficient to represent the corresponding Green's function for a top area of the embankment. This can be done in a way similar to a ray approach to the representation of the field in an ideal waveguide, i.e. by considering the waveguide contribution to the Green's function in question as an infinite sum of Rayleigh waves radiated by imaginary sources with the amplitudes defined by multiples of the reflection coefficient $R_{t}(\alpha)$. Then, for a point source located at $y^{\prime}=0$ and for observation points located in the flat top area inside the embankment, i.e. for $y<|W|$, the resulting Green's function has the form 


$$
\begin{aligned}
G_{z z}(\rho, \omega)= & D(\omega)\left[\left(1 / \sqrt{ } \rho^{(0)}\right) \exp \left(i k_{R} \rho^{(0)}-\gamma k_{R} \rho^{(0)}\right)+\right. \\
& \sum_{n=1}^{\infty}\left(R_{t n}{ }^{(1)}\right)^{n}\left(1 / \sqrt{ } \rho_{n}{ }^{(1)}\right) \exp \left(i k_{R} \rho_{n}{ }^{(1)}-\gamma k_{R} \rho_{n}{ }^{(1)}\right)+ \\
& \left.\sum_{n=1}^{\infty}\left(R_{t n}{ }^{(2)}\right)^{n}\left(1 / \sqrt{ } \rho_{n}{ }^{(2)}\right) \exp \left(i k_{R} \rho_{n}{ }^{(2)}-\gamma k_{R} \rho_{n}{ }^{(2)}\right)\right] .
\end{aligned}
$$

Here $D(\omega)=(1 / 2 \pi)^{1 / 2}(-i \omega) q k_{R}^{1 / 2} k_{t}^{2} \exp (-i 3 \pi / 4) / \mu F^{\prime}\left(k_{R}\right) \quad$ (see also equations (13) and (14)), and $\rho^{(0)}=\left[\left(x-x^{\prime}\right)^{2}+(y)^{2}\right]^{1 / 2}, \quad \rho_{n}{ }^{(1)}=\left[\left(x-x^{\prime}\right)^{2}+(2 W n-y)^{2}\right]^{1 / 2}, \quad \rho_{n}{ }^{(2)}=\left[\left(x-x^{\prime}\right)^{2}+(-2 W n-y)^{2}\right]^{1 / 2}$, $R_{t n}{ }^{(1)}$ and $R_{t n}{ }^{(2)}$ are the values of the reflection coefficient (35) taken for the angles $\alpha=\alpha_{n 1}$ and $\alpha=\alpha_{n 2}$ defined by the expressions $\alpha_{n 1}=\arccos \left[(2 H-y) / \rho_{n}{ }^{(1)}\right]$ and $\alpha_{n 2}=\arccos [(2 H$ $\left.+y) / \rho_{n}{ }^{(2)}\right]$ respectively; other notations are the same as in the previous sections. For points of observation outside the embankment, i.e. for $y>|W|$, the expressions for the Green's function are constructed in a similar way and include also the transmission coefficient $T_{t}(\alpha)$ for Rayleigh waves passing through the boundary between flat top and side areas of the embankment. For shortness, these expressions are not displayed here. The spatial distribution of surface vibration velocity (in arbitrary units) inside and outside the embankment corresponding to the above mentioned Green's function is shown on Fig. 15. Calculations have been made for the frequency $30 \mathrm{~Hz}$ within the area $48 \mathrm{x} 48 \mathrm{~m}^{2}$, the width of the embankment $2 \mathrm{H}$ being equal to $8 \mathrm{~m}$. One can see that the embankment results in redistribution of Rayleigh waves radiated at sliding angles $\alpha$ relative to the embankment's direction. The corresponding Rayleigh waves become trapped and propagate as waveguide modes, rather than as free cylindrically divergent waves.

\section{Fig.15 here}

Substitution of this Green's function into equation (30) in the way described in the previous sections allows calculation of ground vibrations generated by high-speed trains travelling along the embankments. Since the resulting expressions are too bulky and contain triple summation - over sleepers, carriage axles, and imaginary sources, we consider only the simplest example of a single axle load travelling along the embankment at speed which is slightly higher than Rayleigh wave velocity in the ground.

Figure 16 shows the spatial distribution of ground vibration field (in arbitrary units) generated by a single axle load travelling at trans-Rayleigh speed $v=47 \mathrm{~m} / \mathrm{s}$ along the embankment of $8 \mathrm{~m}$ width and with the slop angle $\beta=30^{\circ}$ over a part of a track consisting of 20 sleepers. The area considered is $48 \times 48 \mathrm{~m}^{2}$. Other parameters used in the calculation were: $c_{R}=45 \mathrm{~m} / \mathrm{s}, \sigma=0.25$, and $\rho_{0}=2000 \mathrm{~kg} / \mathrm{m}^{3}$. The track parameters are the same as in the previous figures, and the effect of track bending waves is neglected.

\section{Fig. 16 here}

One can see from Fig. 16 that generated ground vibrations are propagating predominantly along the embankment where their amplitudes are much larger than in the outside area. This demonstrates that the embankments can act as waveguides for generated ground vibration 
boom, reducing the hazardous impact of very intensive vibrations on the built environment. In the same time, the waveguide effects may result in a large increase of ground vibrations inside the embankment. These waveguide-induced additional vibrations of the embankment, which propagate at speed of the train, are expected to amplify the quasi-static bending deflections in the system track-ground accompanying moving axle loads. As a result of this, very large vibrations of the embankment might be observed at train speeds around the velocity of Rayleigh waves, i.e. before achieving the value of track critical velocity.

\section{CONCLUSIONS}

The theory of generating ground vibrations by high-speed trains described in this chapter shows that if train speeds exceed the velocity of Rayleigh surface waves in the supporting soil a ground vibration boom occurs associated with a very large increase in amplitudes of generated vibrations. Crossing the track wave critical velocity results in further increase of generated ground vibrations, albeit not as dramatic as in the case of ground vibration boom.

Recent experimental observations of ground vibration boom generated by Swedish X2000 trains confirm the main predictions of the theory. This implies that Railway-generated ground vibration boom is no longer an exotic theoretical effect. It is a today's reality for high-speed railways crossing soft soil, and so are "supersonic" or "trans-Rayleigh" trains. Builders and operators of high-speed railways must be aware of possible consequences of ground vibration boom, as well as dynamic rail deflections. One can expect that, with the general trend of increasing operating train speeds, this phenomenon will occur in many countries, especially in those with railways built on soft soil.

Problems associated with track-soil critical velocities may take place also for underground trains. In this case the first critical velocity is the velocity of shear bulk elastic waves, and the ground vibration boom for train speeds exceeding this velocity represents a Mach cone of shear waves radiated from the tunnel.

Waveguide effects of the embankments may cause concentration of ground vibration energy radiated by trans-Rayleigh trains. This can reduce ground vibrations outside the embankment. However, the vibrations of the embankment itself may increase significantly. Since these guided vibrations propagate at speed of the train, they may amplify the quasi-static bending deflections in the system track-ground moving with the train.

It is too early on at this stage to foresee how the phenomenon of railway-generated ground vibration boom and its amplification due to track dynamics effects will be reflected in future standards on noise and vibration from high-speed trains. However, one can expect that such an important parameter as Rayleigh wave velocity in the ground for the sites considered will be present in such standards indicating maximal train speeds beyond which excessive ground vibrations can be expected. 


\section{ACKNOWLEDGEMENTS}

The author is grateful to Prof. G. Degrande for useful discussions of part of the material described in this chapter and for drawing the author's attention to some inaccuracies in some of the previous publications.

\section{REFERENCES}

1. REMINGTON, P.J., KURZWEIL, L.G. \& TOWERS, D.A. (1987), "Low-frequency noise and vibrations from trains”, In: Transportation noise. Reference book, Ed. by Paul Nelson, Butterworths, London e.a., Chapter 16.

2. NEWLAND, D.E. \& HUNT, H.E.M. (1991) "Isolation of buildings from ground vibration: a review of recent progress”, Proc. Instn. Mech. Engrs. 205, 39-52.

3. KRYLOV, V.V. (1994) “On the theory of railway-induced ground vibrations”, Journ. de Physique IV, 4, C5-769-772.

4. KRYLOV, V.V. (1995) "Generation of ground vibrations by superfast trains", Applied Acoustics, 44, 149-164.

5. MADSHUS, C. (1997) Public announcement made at the conference "Ground Dynamics and Man-made Processes: Prediction, Design, Measurement”, ICE, London, 20 November 1997.

6. MADSHUS, C. \& KAYNIA, A.M. (1998) "High speed railway lines on soft ground: dynamic behaviour at critical train speed”, Proc. 6th International Workshop on Railway and Tracked Transit System Noise, Ile des Embiez, France, 108-119.

7. KRYLOV, V.V. (1996) "Vibrational impact of high-speed trains. I. Effect of track dynamics”, Journ. Acoust. Soc. Am., 100 (5), 3121-3134; erratum (1997), 101(6), 3810.

8. KRYLOV, V.V. (1997) "Spectra of low-frequency ground vibrations generated by highspeed trains on layered ground”, Journ. Low Frequency Noise and Vibr., 16(4), 257-270.

9. KRYLOV, V.V. (1998) "Effect of track properties on ground vibrations generated by high-speed trains”. Acustica-acta acustica, 84(1), 78-90.

10. TAKEMIYA, H. (1998) "Lineside ground vibrations induced by high-speed train passage”, Proc. Of the Workshop on Effect of High-Speed Vibration on Structures and Equipment”, Tainan, Taaiwan, 43-49.

11. DEGRANDE, G., \& SCHILLEMANS, L. (1998) "Free field vibrations during the passage of HST", Proc. ISMA 23, Noise and Vibration Engineering, Ed. P.Sas, Leuven, Belgium, Vol. 3, p. 1563-1570. 
12. PETYT, M. \& JONES, C.J.C. (1999) "Modelling of ground-borne vibrations from railways”, Proc. 4th European Conference on Structural Dynamics (EURODYN’99), Prague, Czech Republic, 79-87.

13. SHENG, X., JONES, C.J.C. \& PETYT, M. (1999), "Ground vibration generated by a load moving along a railway track”, Journal of Sound and Vibration, 228(1), 129-156.

14. WOLDRINGH, R.F. \& NEW, B.M. "Embankment design for high speed trains on soft soils”, Proc. 12th Eur. Conf. Soil Mech and Geotechn. Eng., "Geotechnical Engineering for Transportation Infrastructure”, Amsterdam, The Netherlands, 7-10 June 1999, Eds. F.B.J. Barends et al., A.A. Balkema, Rotterdam, Vol. 3, p. 1703-1712.

15. ADOLFSSON, K., ANDREASSON, B., BENGTSSON, P.-E., \& ZACKRISSON, P. (1999) "High speed train X2000 on soft organic clay - measurements in Sweden", Proc. 12th Eur. Conf. Soil Mech and Geotechn. Eng., "Geotechnical Engineering for Transportation Infrastructure”, Amsterdam, The Netherlands, 7-10 June 1999, Eds. F.B.J. Barends et al., A.A. Balkema, Rotterdam, Vol. 3, p. 1713-1718.

16. HEELIS, M.E., COLLOP, A.C., DAWSON, A.R., CHAPMAN, D.N. \& KRYLOV, V.V. (1999) "Transient effects of high speed trains crossing soft soil”, Proc. 12th Eur. Conf. Soil Mech and Geotechn. Eng., "Geotechnical Engineering for Transportation Infrastructure”, Amsterdam, The Netherlands, 7-10 June 1999, Eds. F.B.J. Barends et al., A.A. Balkema, Rotterdam, Vol. 3, p. 1809-1814.

17. KRYLOV, V.V., DAWSON, A.R., HEELIS, M.E. \& COLLOP, A.C. (2000) Rail movement and ground waves caused by high-speed trains approaching track-soil critical velocities. Proc. IME: Journ. Rail and Rapid Transit (Accepted for publication).

18. KRYLOV, V.V. \& FERGUSON, C.C. (1994) "Calculation of low-frequency ground vibrations from railway trains”, Applied Acoustics, 42, 199-213.

19. FRYBA, L. (1973) “Vibration of solids and structures under moving loads", Noordhoff, Groningen, The Netherlands.

20. BROCKLEY, C.A. (1992), “The influence of track support structure and locomotive traction characteristics on short wavelength corrugations”, Wear, 153, 315-322.

21. EWING, W.M., JARDETZKY, W.S. \& PRESS, F. (1957) "Elastic waves in layered media”, McGraw-Hill, New York.

22. GRAFF, K.F. (1975), “Wave motion in elastic solids”, Clarendon Press, Oxford.

23. GUTOVSKI, T.G. \& DYM, C.L. (1976) "Propagation of ground vibration: a review”, Journ. of Sound and Vibration, 49, 179-193.

24. BIRYUKOV, S.V., GULYAEV, Y.V., KRYLOV, V.V. \& PLESSKY, V.P. (1995) "Surface acoustic waves in inhomogeneous media", Springer Series on Wave Phenomena, Vol. 20, Springer-Verlag, Berlin, Heidelberg et al. - 390 pp. 
25. COLE, J. \& HUTH, J. (1958) “Stresses produced in a half plane by moving loads", Journ. Appl. Mech., 25, 433-436.

26. KRYLOV, V.V. (1995) Low-frequency ground vibrations from underground trains. Journ. Low Frequency Noise and Vibr., 14, No 1, 55-60.

27. LIN, Q. \& KRYLOV, V.V. (2000) Effect of tunnel diameter on ground vibrations generated by underground trains. Journ. of Low Frequency Noise, Vibration and Active Control (Accepted for publication).

\section{FIGURE CAPTIONS}

Fig. 1. Geometry of track and train - (a) and the wheel-axle pressure mechanism of generating ground vibrations - (b).

Fig. 2. Spectra of vertical forces applied from each sleeper to the ground (in Ns) for three values of train speed $v$ corresponding to the cases $v<c_{R}, c_{R}<v<c_{\min }$, and $v>c_{\text {min }}$ respectively: $v=20 \mathrm{~m} / \mathrm{s}$ (curve P1), $50 \mathrm{~m} / \mathrm{s}$ (curve P2), and $70 \mathrm{~m} / \mathrm{s}$ (curve P3); frequency $\mathrm{f}$ is in $\mathrm{Hz}$.

Fig. 3. Some typical profiles of layered ground.

Fig. 4. Approximate analytical functions describing frequency-dependent Rayleigh wave velocities $c_{R}(\omega)$ for monotonous ground profiles:

$c d 1=(c 1-c 2) \exp \left(-\alpha^{\prime} 1 f\right)+c 2, \quad c d 2=(c 1-c 2) \exp \left(-\alpha^{\prime} 2 f\right)+c 2$,

$c d 3=(c 2-c 1) \exp \left(-\alpha^{\prime} 1 f\right)+c 1, c d 4=(c 2-c 1) \exp \left(-\alpha^{\prime} 2 f\right)+c 1$;

$\alpha^{\prime} 1=0.1$ and $\alpha^{\prime} 2=10$.

Fig. 5. Spatial distributions of the surface vertical displacements (in arbitrary units) generated at the frequency component $f=31.4 \mathrm{~Hz}$ by a single axle load moving through a small part of a track consisting of 10 sleepers; the results are shown for sub-Rayleigh speed - (a) and for trans-Rayleigh speed - (b).

Fig. 6. Spectra of ground vibration velocity (in linear units, relative to the reference level of $10^{-9} \mathrm{~m} / \mathrm{s}$ ) for a single axle load moving along the track at speeds from $10 \mathrm{~m} / \mathrm{s}$ to 320 $\mathrm{m} / \mathrm{s}$ on the surface of a homogeneous (a) and layered (b) half space. The results are shown in the form of surface graphs for the frequency band $0-50 \mathrm{~Hz}$. Mesh: $\Delta v=10$ $\mathrm{m} / \mathrm{s}$ and $\Delta F=1 \mathrm{~Hz}$. 
Fig. 7. Ground vibration spectra (in $\mathrm{dB}$, relative to the reference level of $10^{-9} \mathrm{~m} / \mathrm{s}$ ) generated by complete $\mathrm{TGV}$ or Eurostar trains travelling on homogeneous ground at subRayleigh and trans-Rayleigh speeds (respectively: $v=50 \mathrm{~km} / \mathrm{h}-$ curve Vz1 and $v=$ $500 \mathrm{~km} / \mathrm{h}$ - curve Vz2) and on layered ground at the same speeds (curves Vz3 and Vz4 respectively).

Fig. 8. Ground vibration spectra (in $\mathrm{dB}$, relative to the reference level of $10^{-9} \mathrm{~m} / \mathrm{s}$ ) generated by TGV trains comprising $N=5$ equal carriages for three values of train speed $v$ corresponding to the cases $v<c_{R}, c_{R}<v<c_{\min }$, and $v>c_{\min }$ respectively: $v=20$ $\mathrm{m} / \mathrm{s}$ (curve v1), $50 \mathrm{~m} / \mathrm{s}$ (curve v2) and $70 \mathrm{~m} / \mathrm{s}$ (curve v3); ); frequency $\mathrm{f}$ is in $\mathrm{Hz}$.

Fig. 9. Ground vibration velocities averaged in the frequency range of $0-50 \mathrm{~Hz}$ (in $10^{-5} \mathrm{~m} / \mathrm{s}$ ) for TGV trains comprising $N=5$ equal carriages; the resulting amplitudes as functions of train speed $v$ (in $\mathrm{m} / \mathrm{s}$ ) are shown for two values of track critical velocity: $-c_{\min }=65 \mathrm{~m} / \mathrm{s}$ (curve V1a) and $c_{\min }=10000 \mathrm{~m} / \mathrm{s}$ (curve V2a) (the latter very large value of $c_{\min }$ describes the hypothetical case when track dynamics effects can be ignored).

Fig. 10. Trains travelling in underground tunnels.

Fig. 11. Ground vibration amplitudes (in $\mathrm{dB}$, relative to the reference level of $10^{-9} \mathrm{~m} / \mathrm{s}$ ) generated by underground TGV trains comprising $N=5$ equal carriages at frequency $f=15 \mathrm{~Hz}$ as functions of the tunnel depth $H$ (in $\mathrm{m}$ ); the results are shown for two values of train speed: $v=13,8 \mathrm{~m} / \mathrm{s}$ (conventional speed) and $v=80 \mathrm{~m} / \mathrm{s}$ (trans-shear speed for the ground considered); the vertical components of ground vibration velocity on the ground surface are indicated as Vz1 and Vz2 respectively for $v=$ $13.8 \mathrm{~m} / \mathrm{s}$ and $v=80 \mathrm{~m} / \mathrm{s}$. The corresponding separate contributions of longitudinal and shear elastic waves are shown as VzL1, VzS1 and VzL2, VzS2.

Fig. 12. Ground vibration amplitudes (in $\mathrm{dB}$, relative to the reference level of $10^{-9} \mathrm{~m} / \mathrm{s}$ ) generated by underground TGV trains comprising $N=5$ equal carriages at frequency $f=15 \mathrm{~Hz}$ as functions of the observation distance from the track $Y_{0}$ (in $\mathrm{m} \mathrm{);} \mathrm{the}$ results are shown for two values of train speed: $v=13,8 \mathrm{~m} / \mathrm{s}$ (conventional speed) and $v=80 \mathrm{~m} / \mathrm{s}$ (trans-shear speed for the ground considered); the vertical components of ground vibration velocity on the ground surface are indicated as Vz01 and Vz02 respectively for $v=13.8 \mathrm{~m} / \mathrm{s}$ and $v=80 \mathrm{~m} / \mathrm{s}$.

Fig. 13. Ground vibration spectra (in $\mathrm{dB}$, relative to the reference level of $10^{-9} \mathrm{~m} / \mathrm{s}$ ) generated by underground TGV trains comprising $N=5$ equal carriages for two values of train speed: $v=13,8 \mathrm{~m} / \mathrm{s}$ (conventional speed) and $v=80 \mathrm{~m} / \mathrm{s}$ (trans-shear speed for the ground considered) - curves Vz1 and Vz2 respectively; for comparison, the ground vibration spectra are shown generated by the same train travelling above the ground (curves Vz1R and Vz2R respectively).

Fig. 14. Amplitude and phase of the truncated Rayleigh wave reflection coefficient $R_{t}(\alpha)$ from the boundary between two surfaces crossing at the angle $\Theta=150^{\circ}\left(\beta=30^{\circ}\right)$ as functions of the incidence angle $\alpha$. 
Fig. 15. Spatial distribution of the surface vertical displacements (in arbitrary units) generated at the frequency component $f=30 \mathrm{~Hz}$ by a single axle load moving over a single sleeper placed on the embankment (the Greens function for the system considered); calculations have been made within the surface area $48 \times 48 \mathrm{~m}^{2}$ for the width of the embankment $2 \mathrm{~W}=8 \mathrm{~m}$.

Fig. 16. Spatial distribution of the surface vertical displacements (in arbitrary units) generated at the frequency component $f=30 \mathrm{~Hz}$ by a single axle load moving at trans-Rayleigh speed $v=127 \mathrm{~m} / \mathrm{s}$ over a part of the track consisting of 20 sleepers placed on the embankment; calculations have been made within the surface area $48 \times 48 \mathrm{~m}^{2}$ for the width of the embankment $2 W=8 \mathrm{~m}$. 
$a$
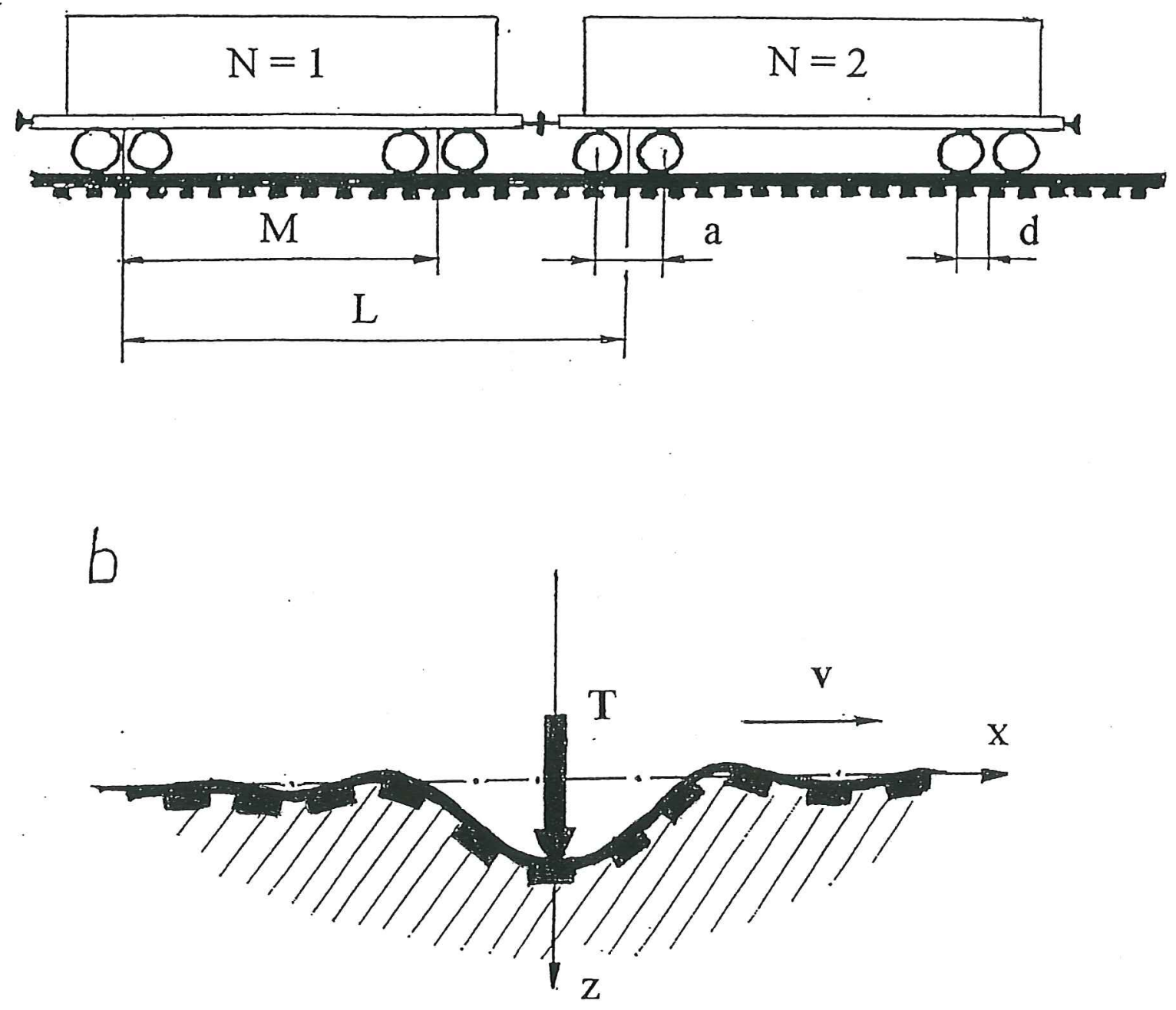

Fig. 1 


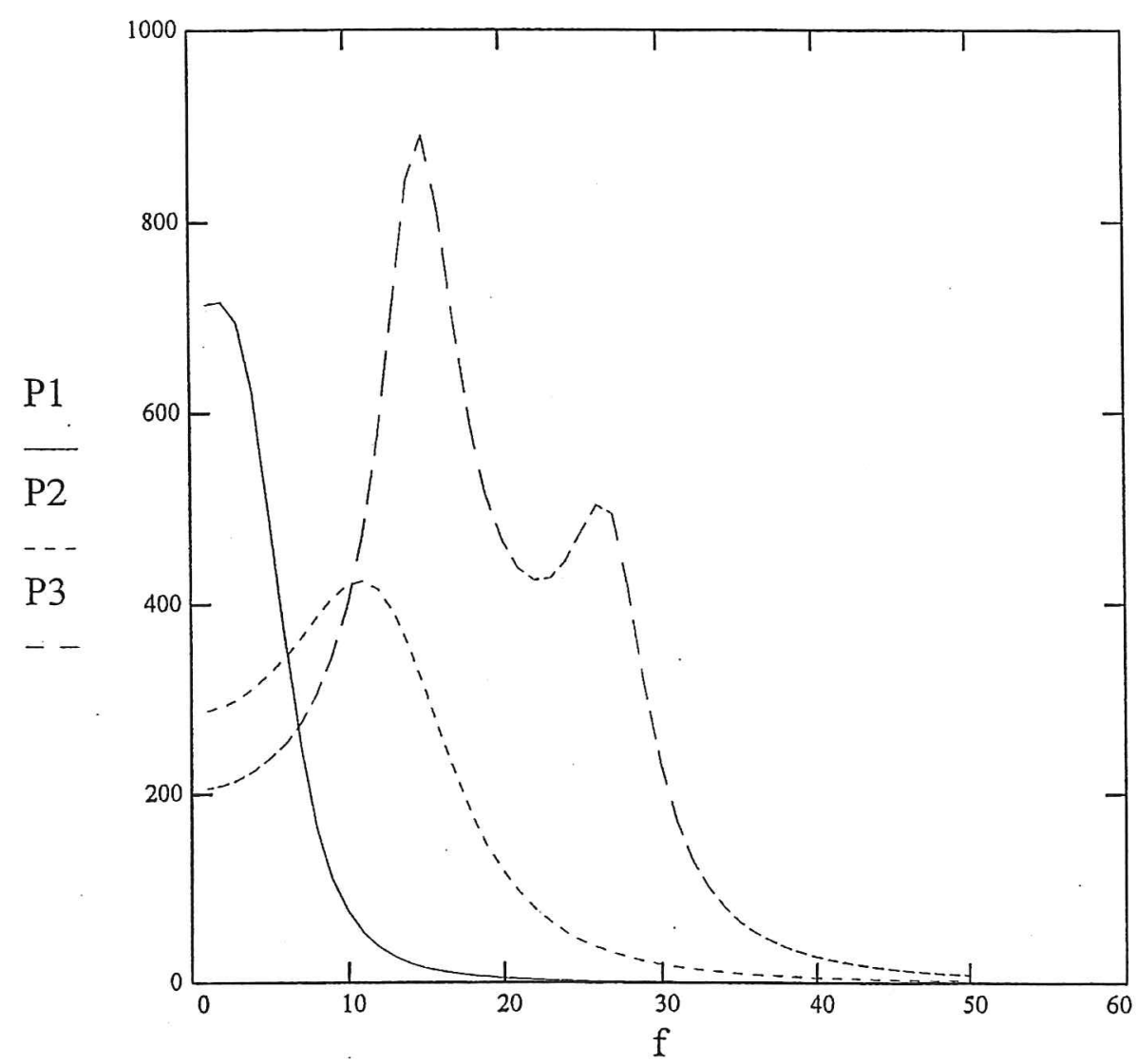

Fig. 2 
(a)

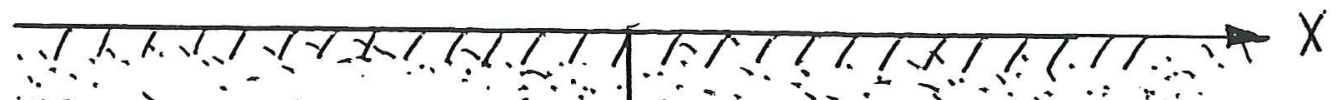
$\because \lambda_{1} \mu_{1} \rho_{01}, \therefore=1, \cdots$

$\lambda_{2} \mu_{2} \rho_{\circ 2}$

z

(b)
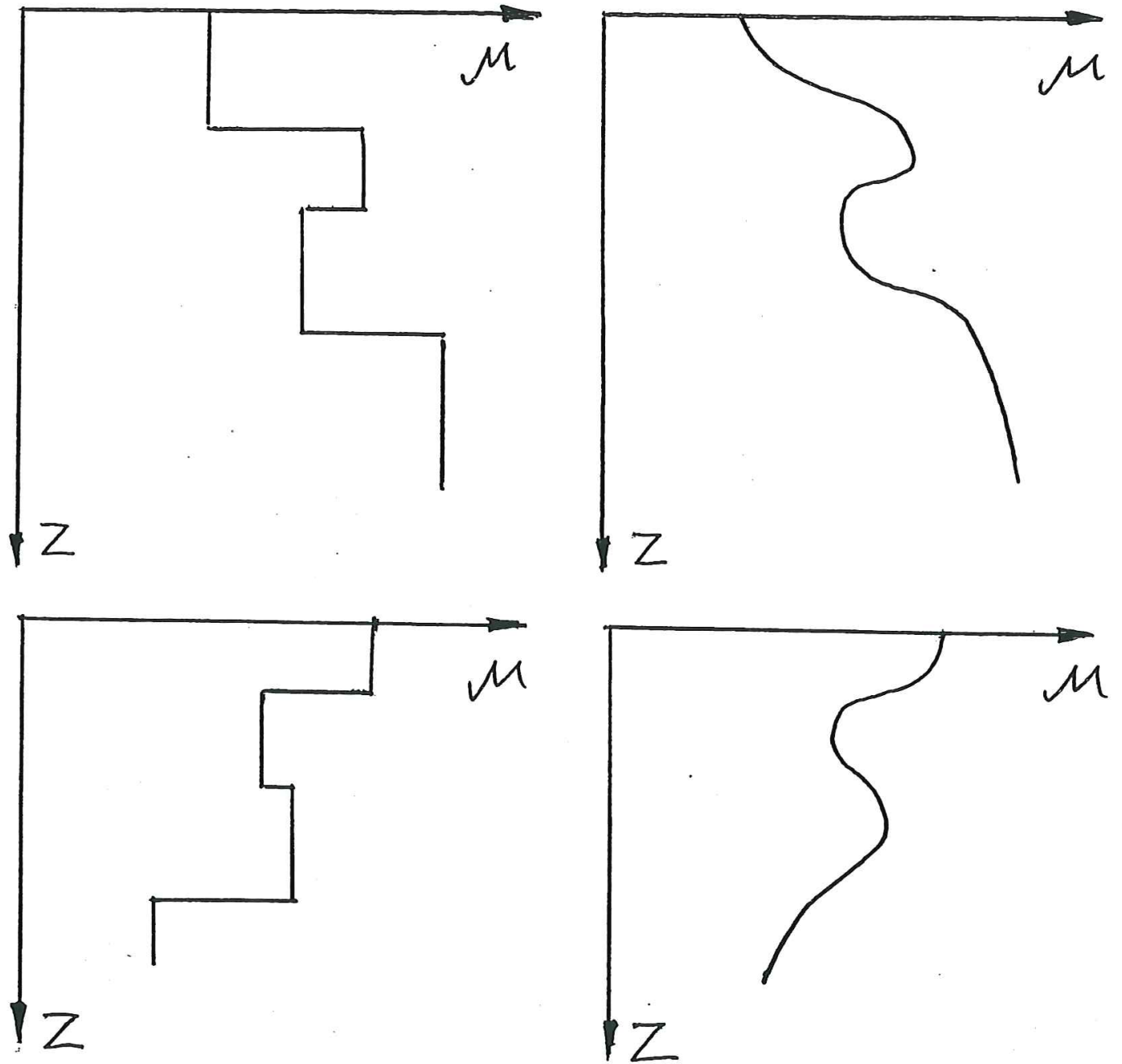

Fig. 3 


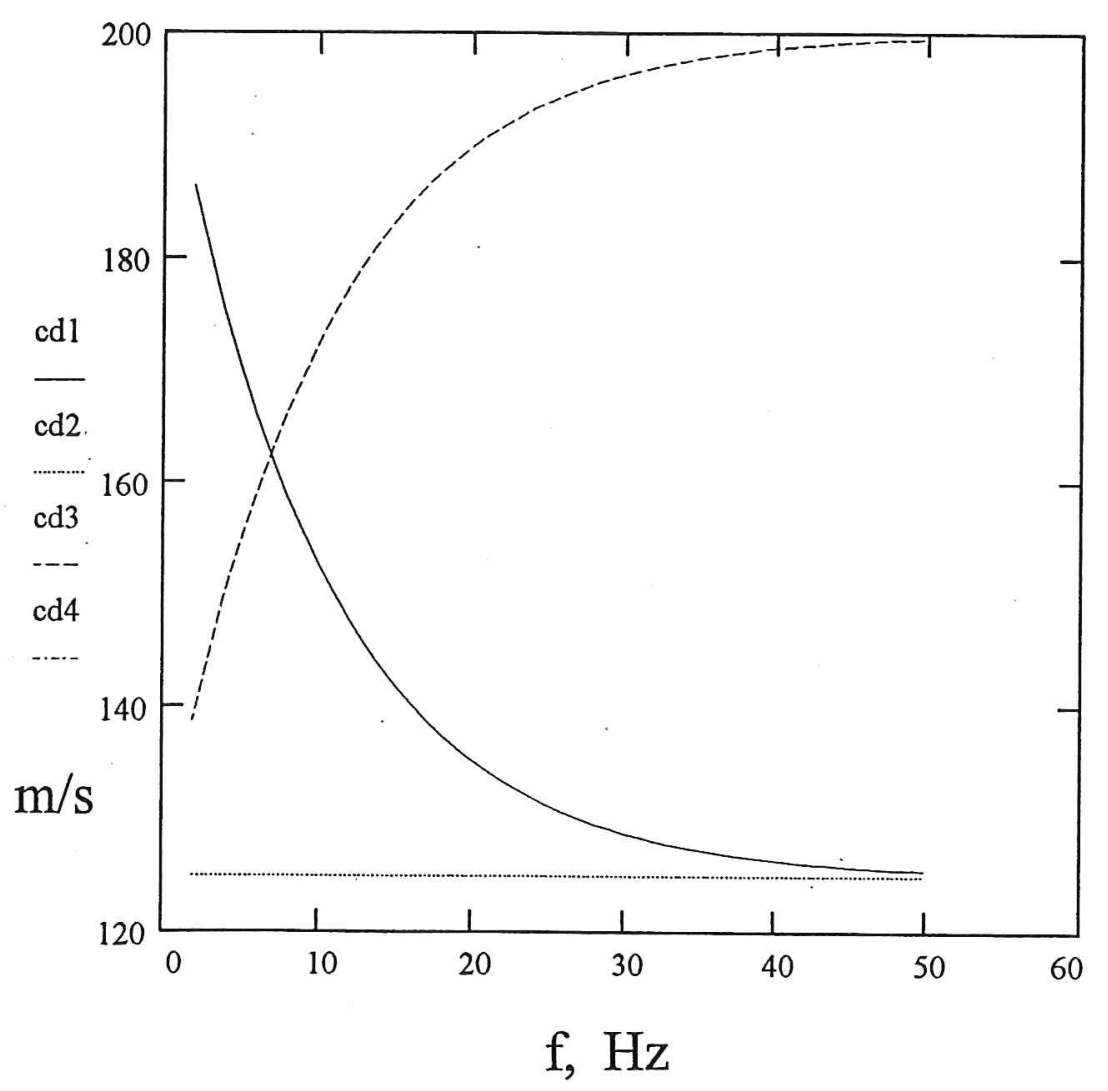

Fig. 4 

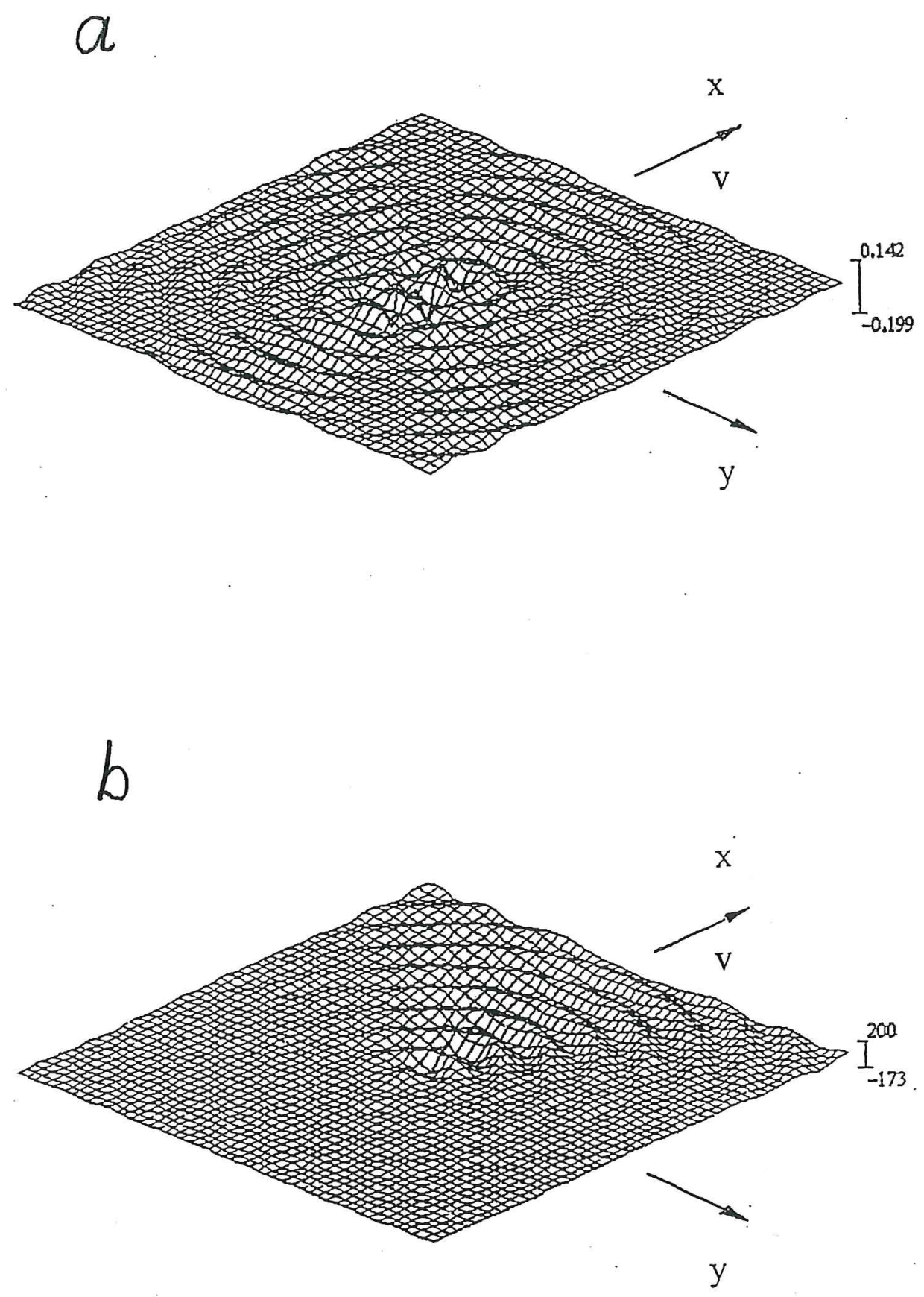

Fig. 5 

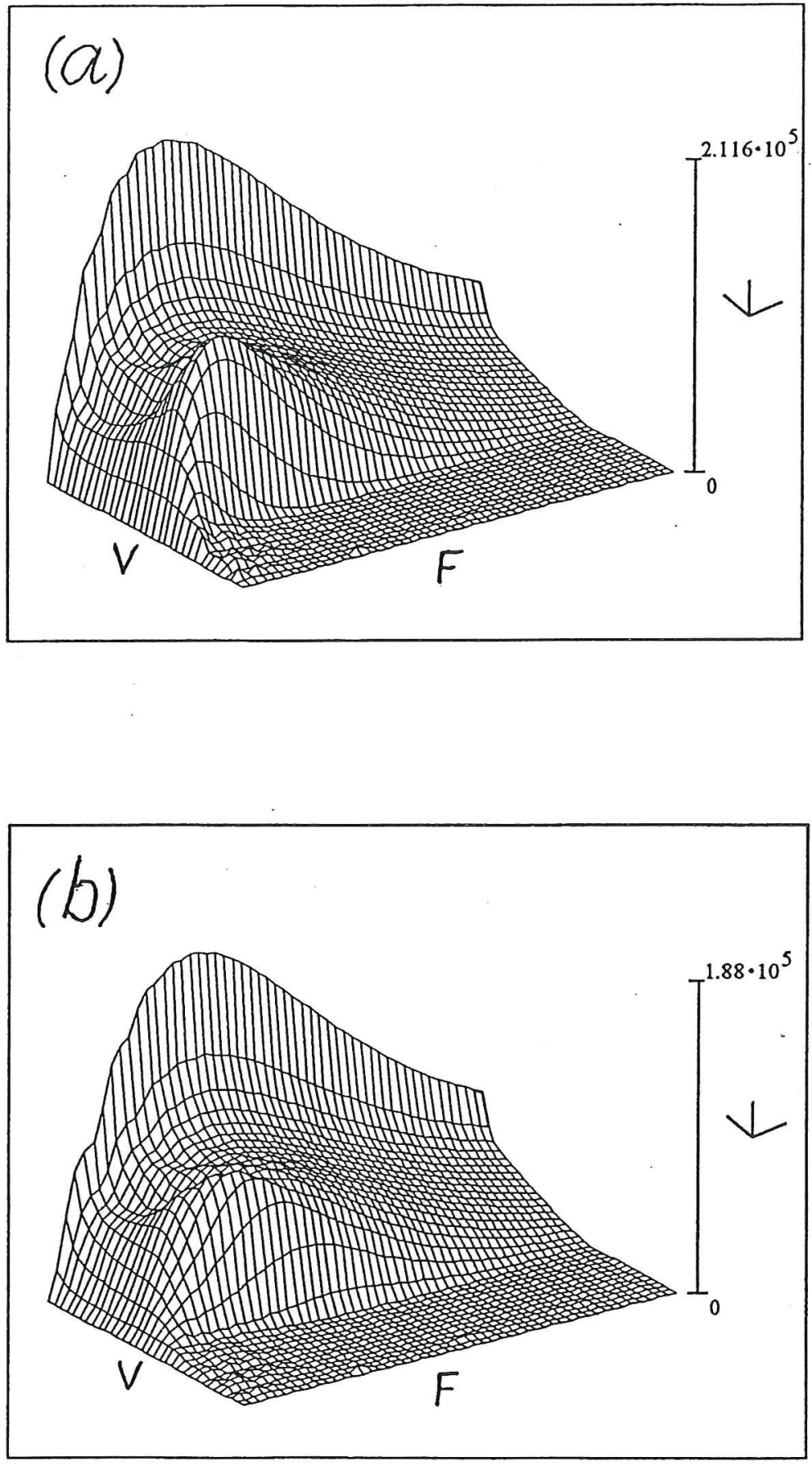

$$
\text { Fig. } 6
$$




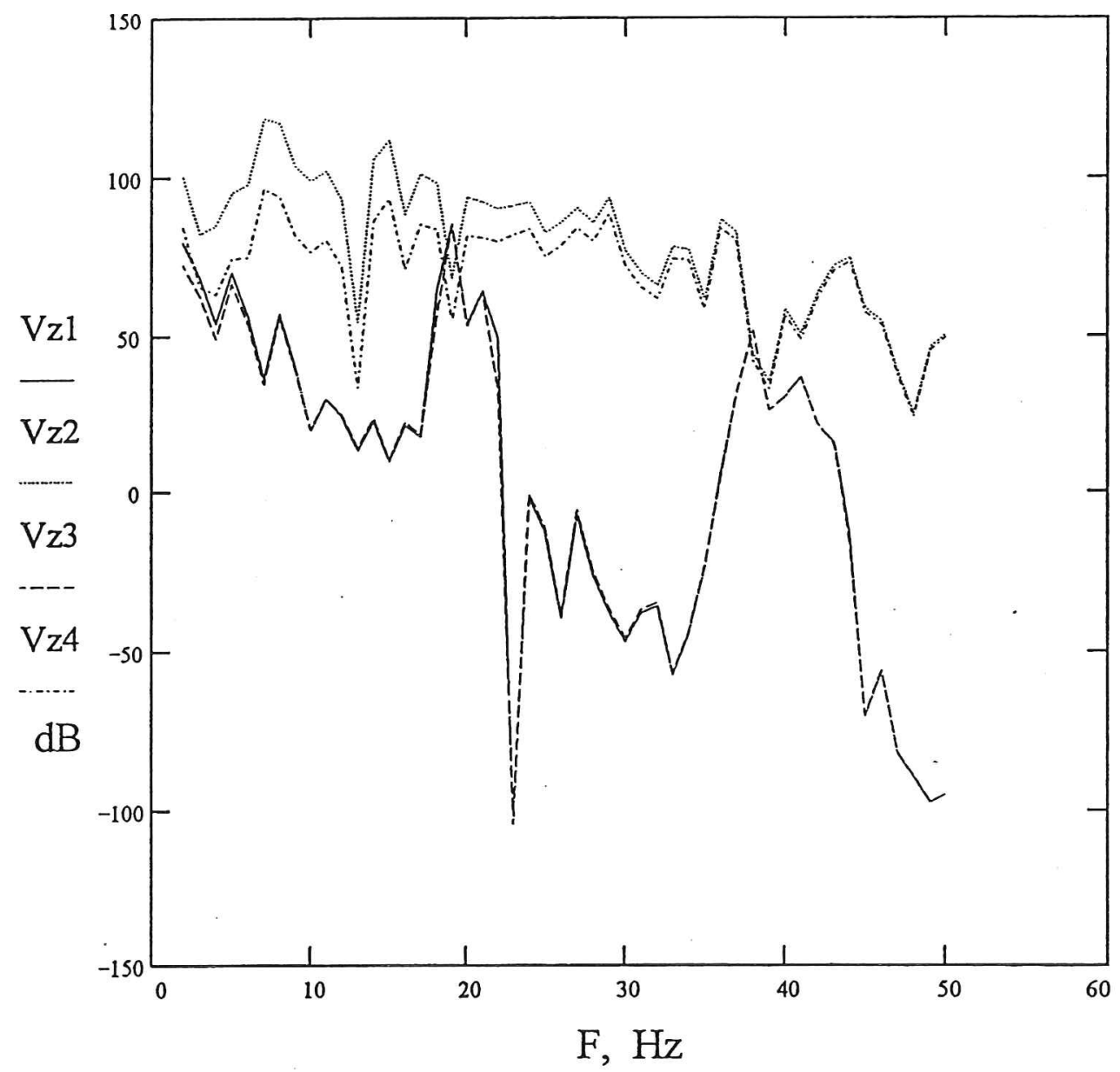

Fig. 7 


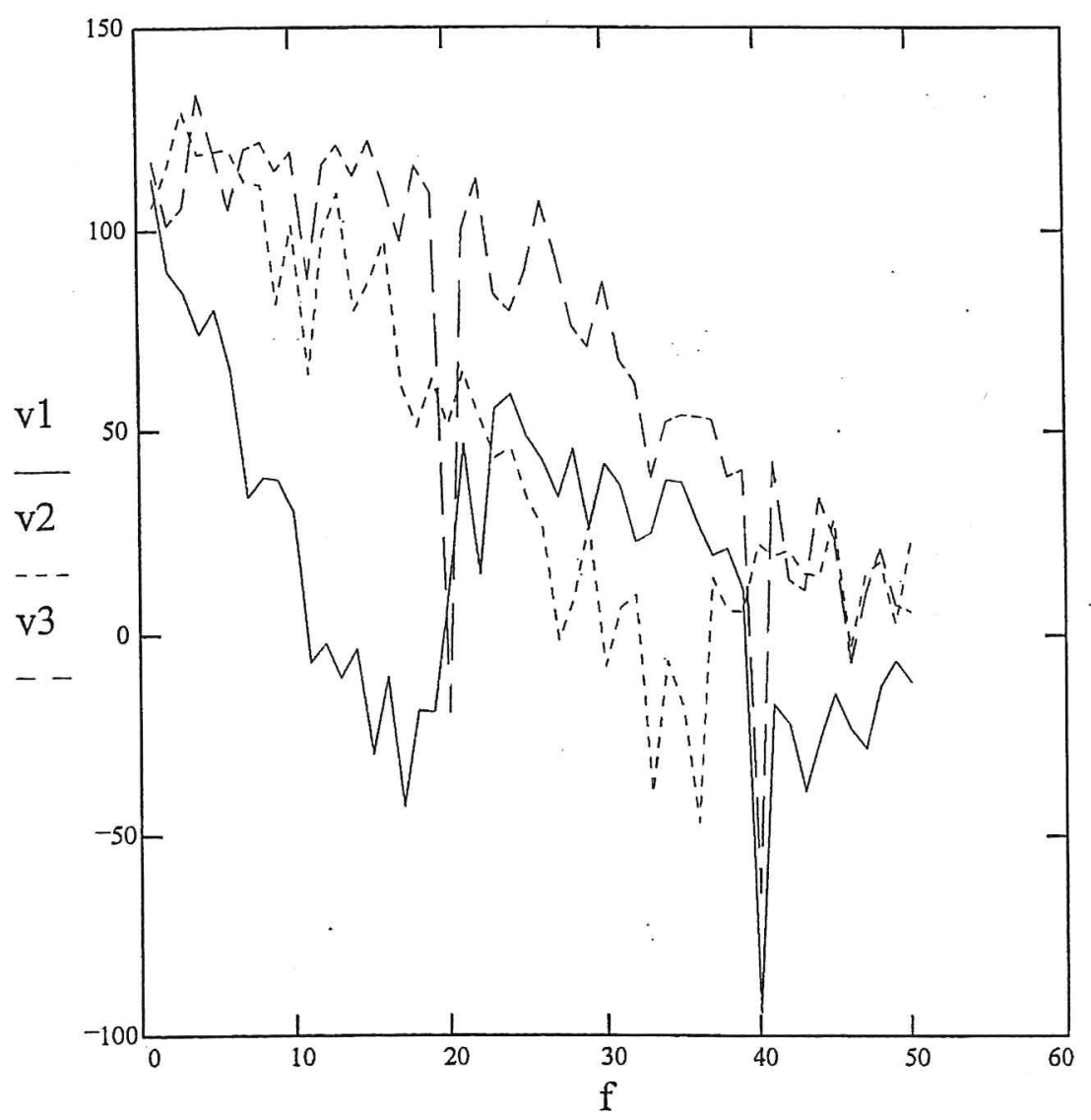

\section{Fig. 8}




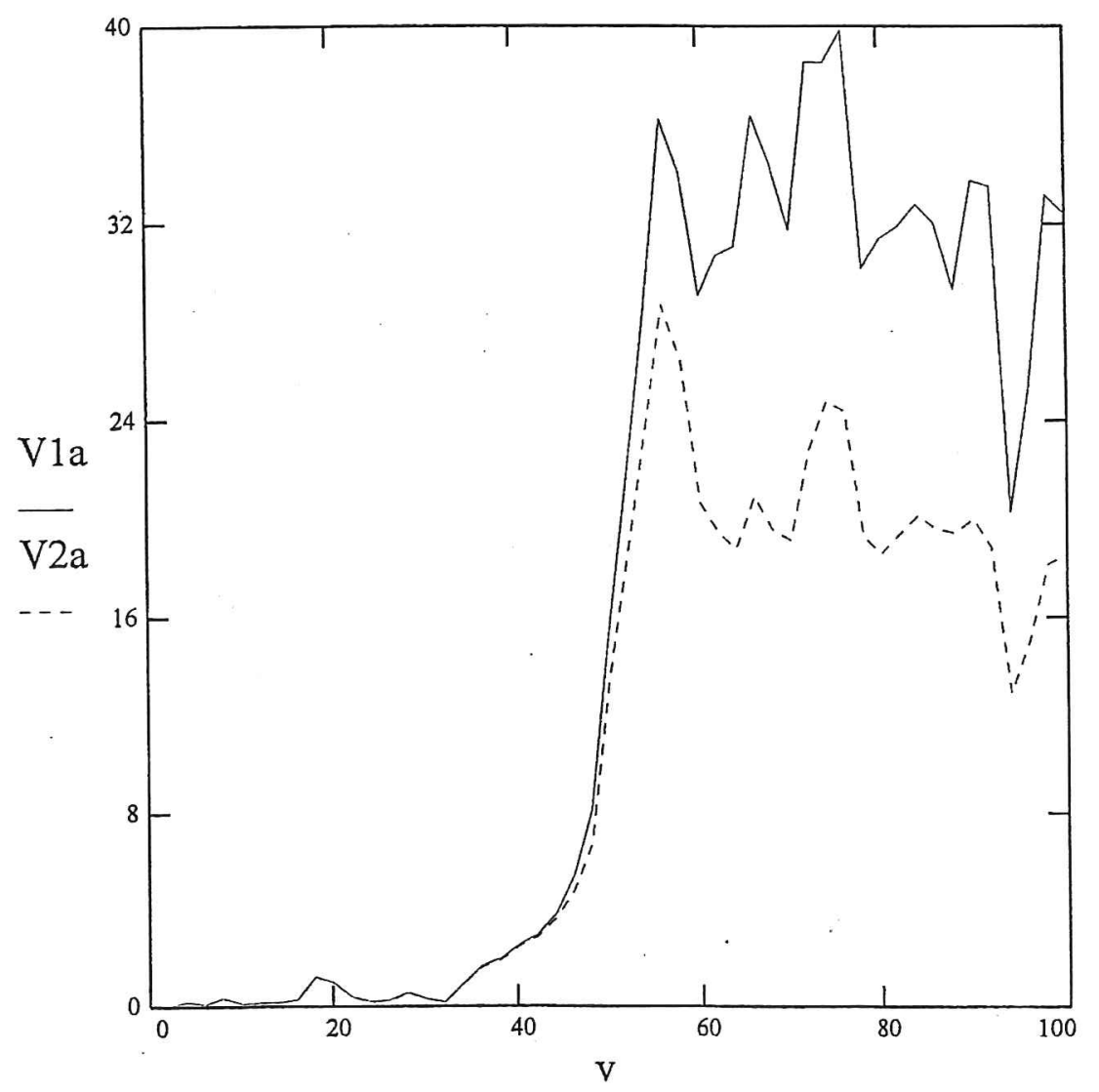

Fig. 9 


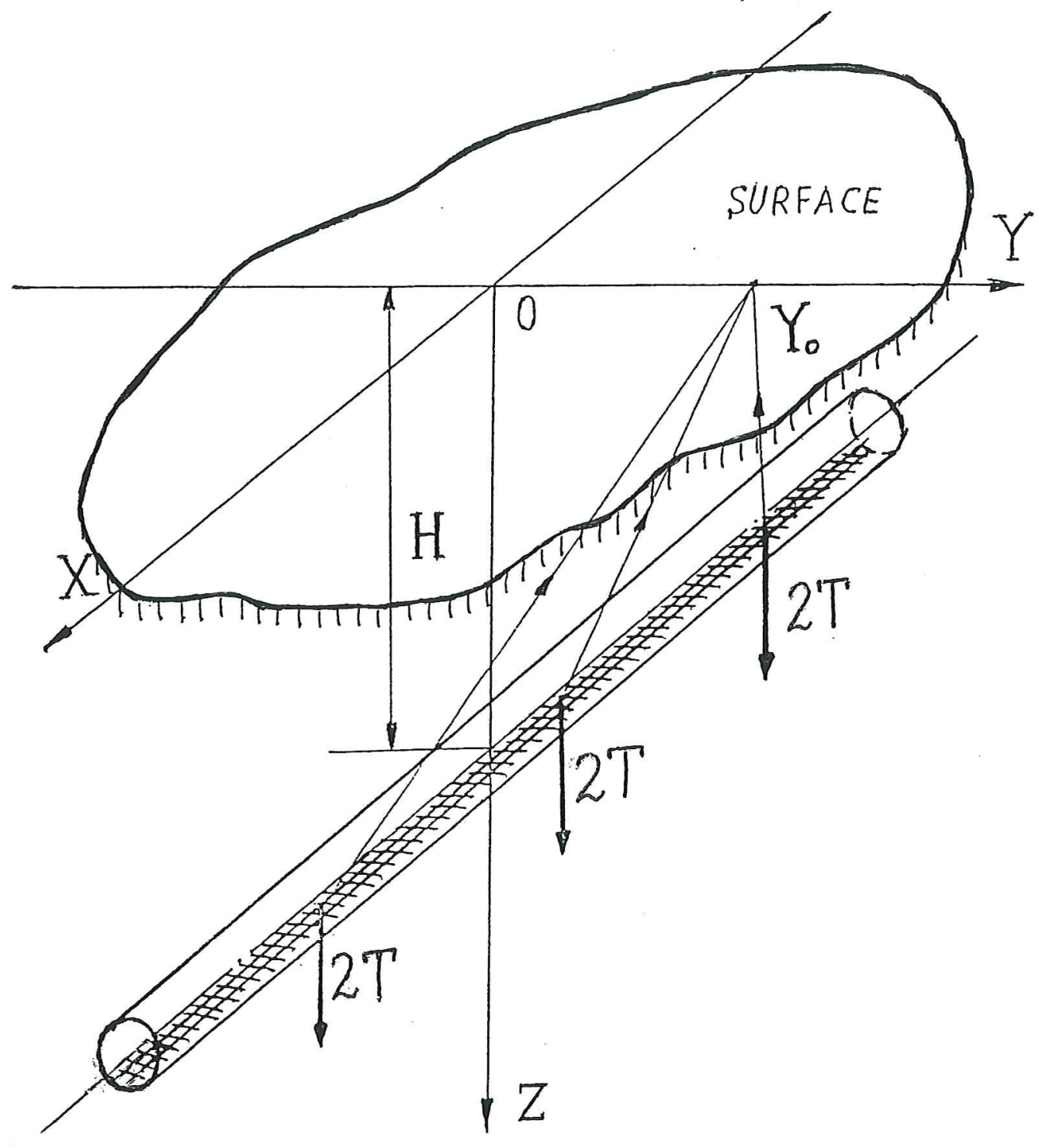

Fig. 10 


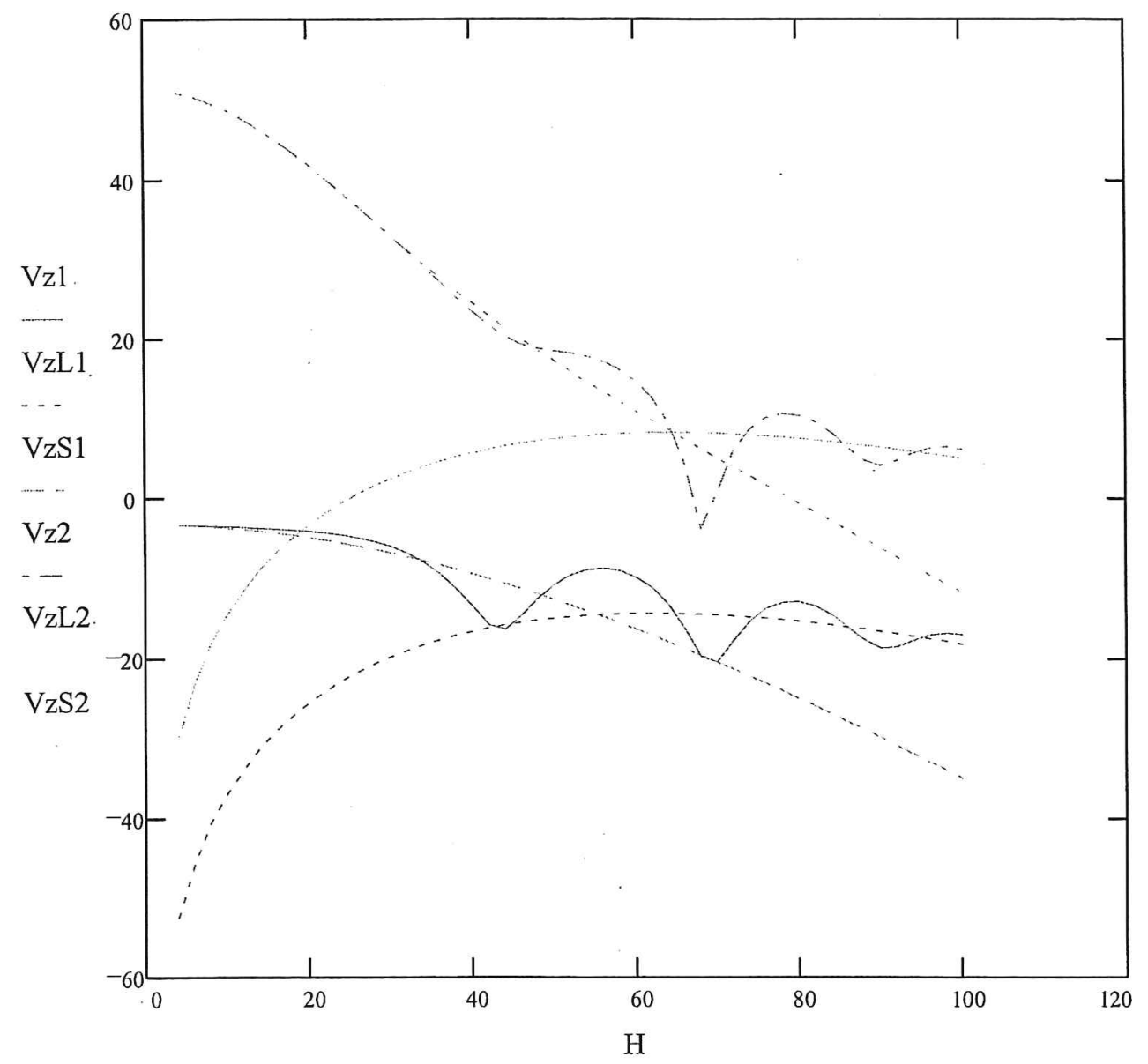

Fig. 11 


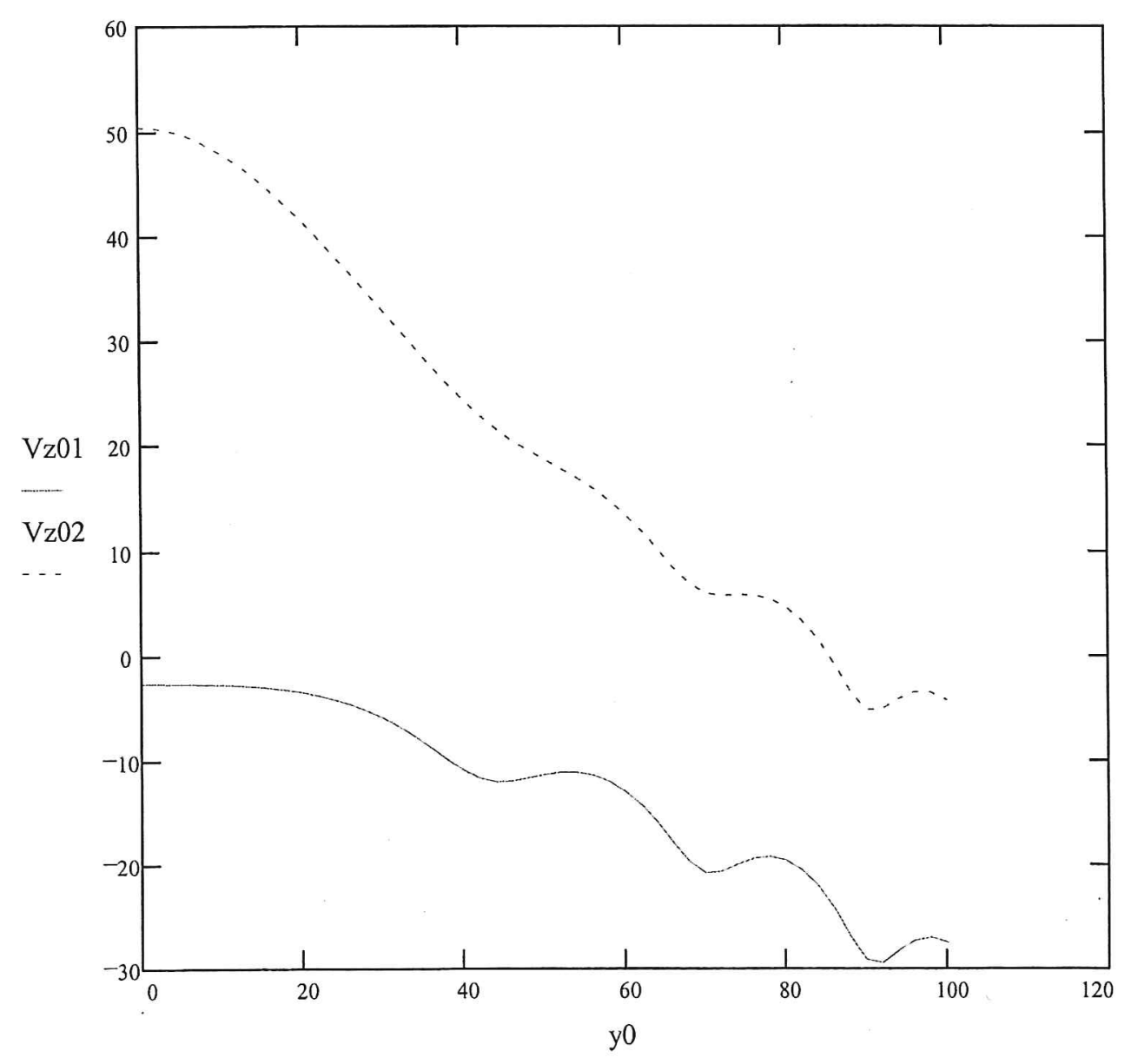

Fig. 12 


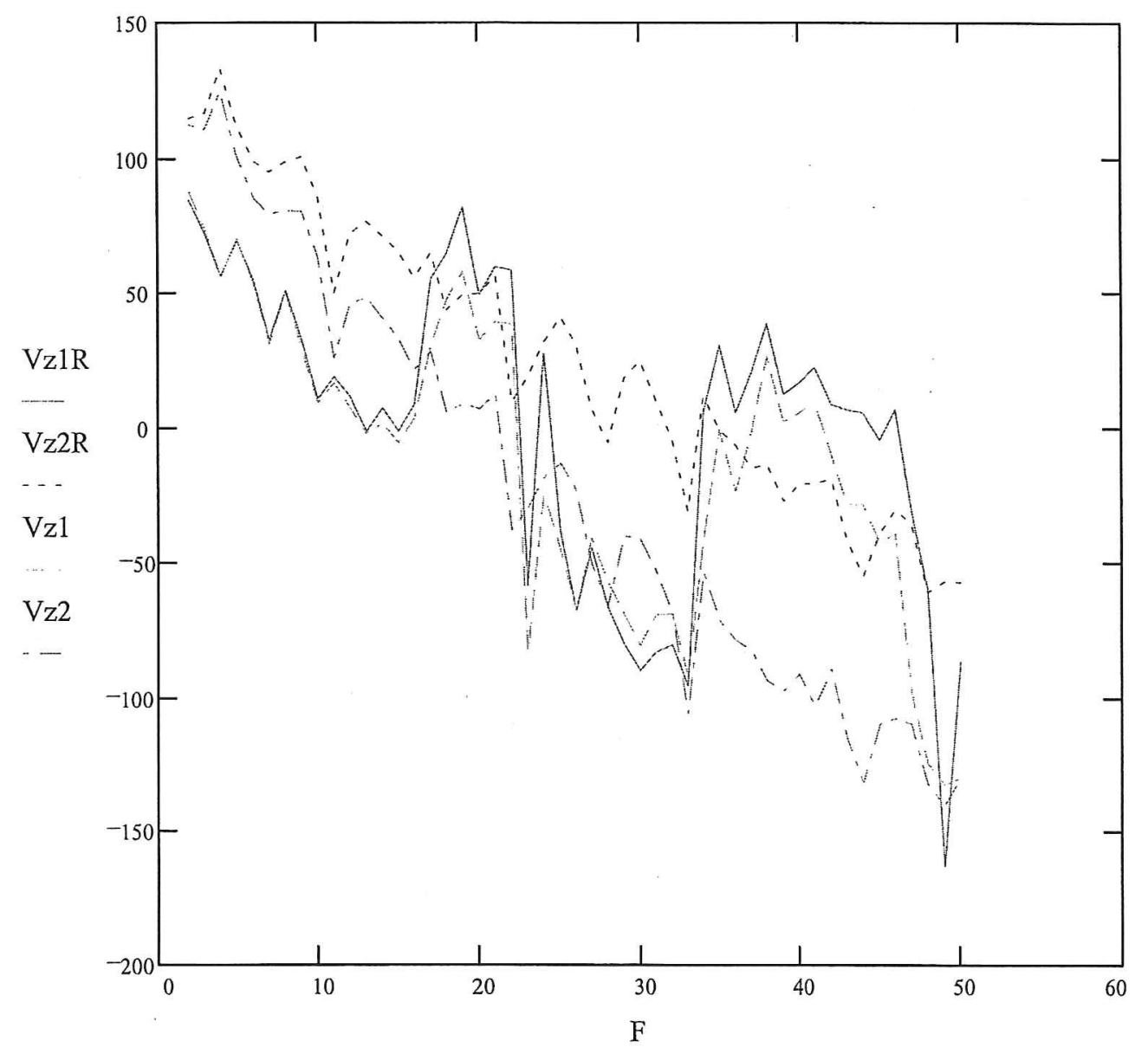

$$
\text { Fig. } 13
$$



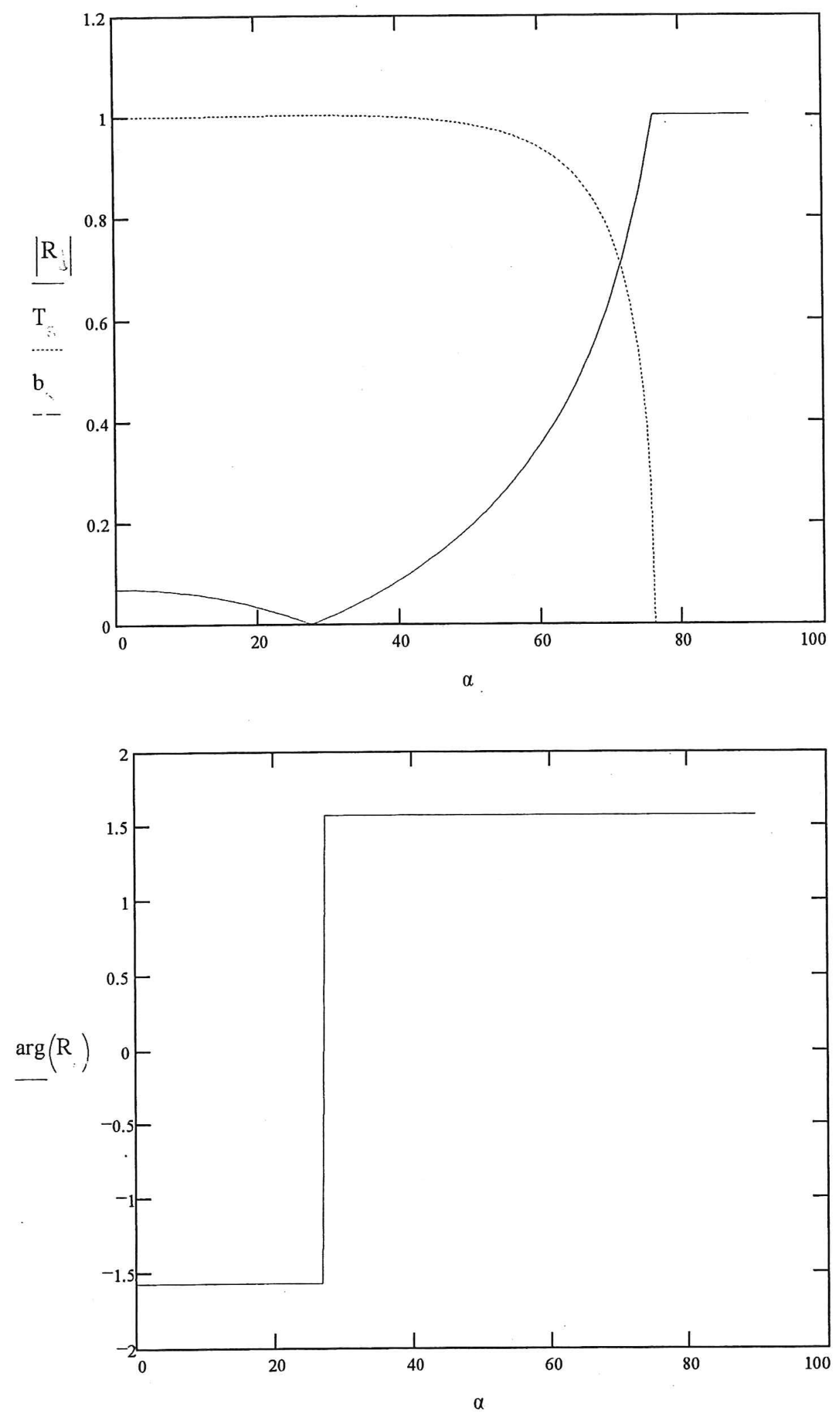

Fig. 14 


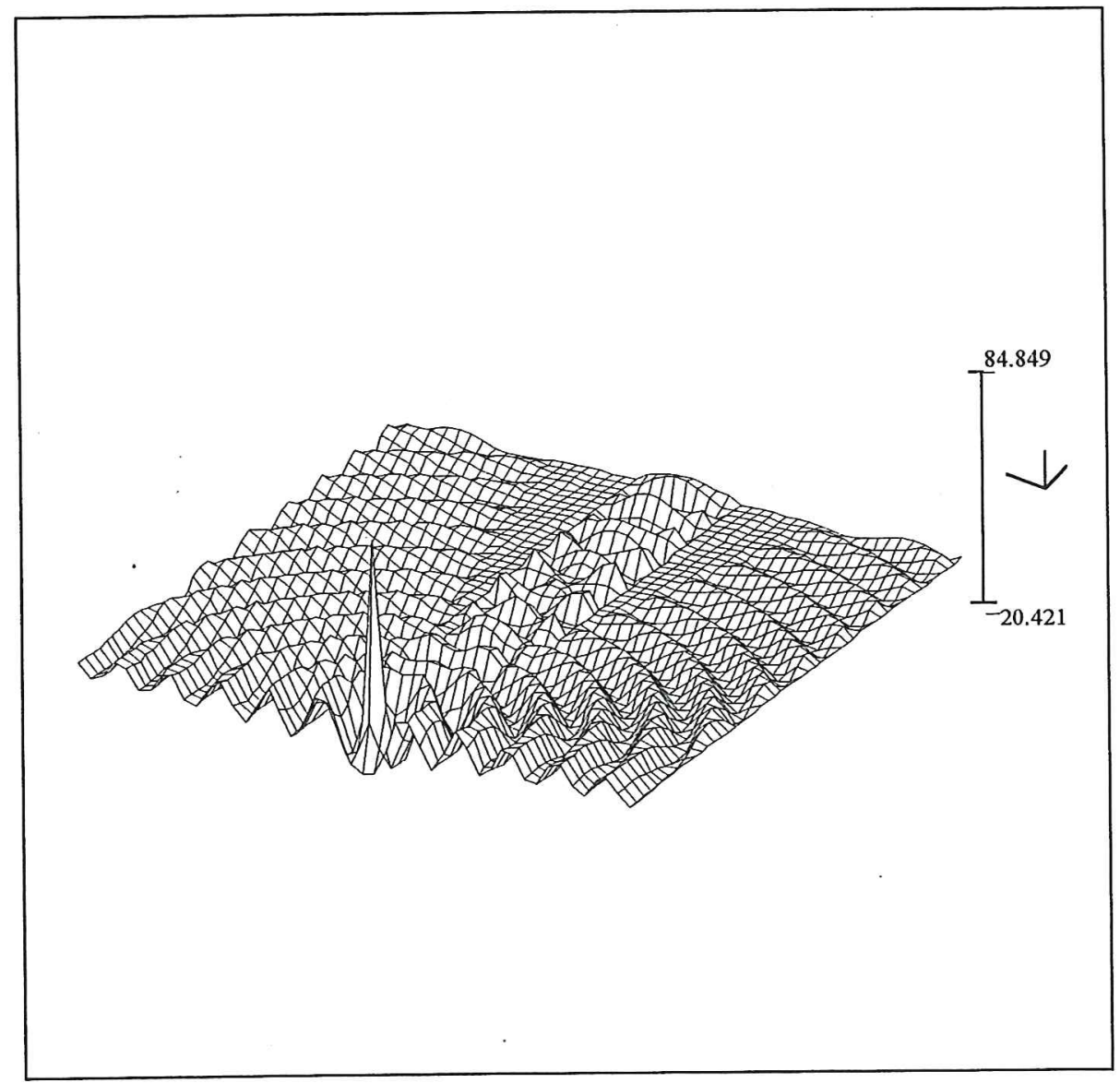

Fig. 15 


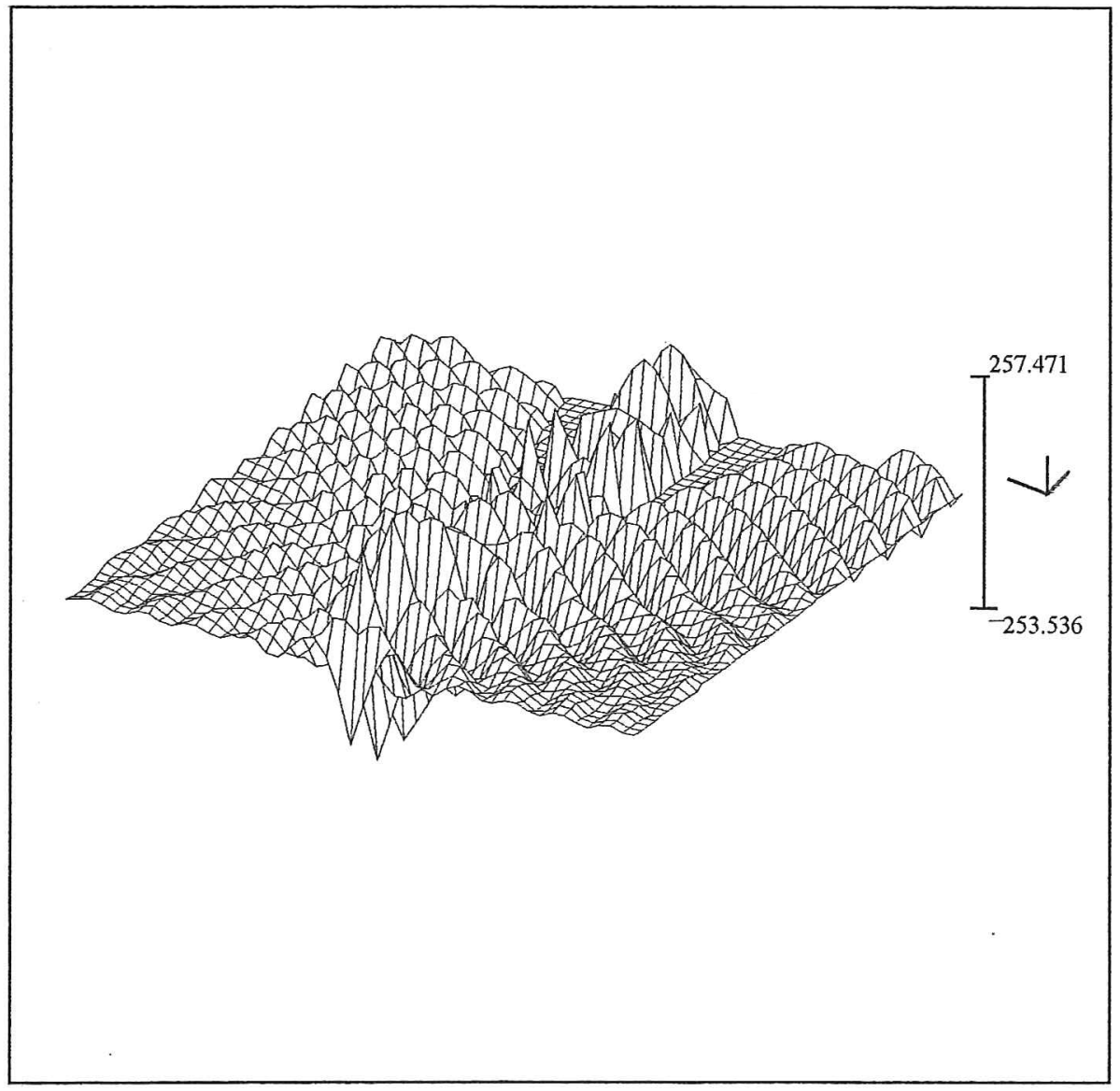

Fig. 16 